\title{
Metamaterials with negative Poisson's ratio and non-positive thermal expansion
}

\author{
L. Ai, X.-L. Gao* \\ Department of Mechanical Engineering, Southern Methodist University, \\ P. O. Box 750337, Dallas, TX 75275-0337, USA
}

\begin{abstract}
Four metallic metamaterials with tailorable mechanical properties are designed using bimaterial star-shaped re-entrant planar lattice structures, which do not involve pins, adhesive, welding or pressure-fit joints and can be fabricated through laser-based additive manufacturing. Three length parameters, one angle parameter and three material combinations are used as adjustable design parameters to explore structure-property relations. For each of the four designed metamaterials, the effects of the design parameters on the Poisson's ratio (PR), coefficient of thermal expansion (CTE), Young's modulus and relative density are systematically investigated using unit cell-based finite element simulations that incorporate periodic boundary conditions. It is found that the bi-material lattice structures can be tailored to obtain 3-D printable metallic metamaterials with positive, near-zero or negative PR and CTE together with an uncompromised Young's modulus. In particular, it is shown that metamaterial \# 1 can exhibit both a negative PR and a non-positive CTE simultaneously. These metallic metamaterials can find applications in structures or devices such as antennas and precision instruments to reduce thermomechanical stresses and extend service lives.
\end{abstract}

Keywords: Metamaterial, re-entrant lattice structure, auxetic material, near-zero thermal expansion, negative Poisson's ratio, additive manufacturing, Young's modulus, relative density

\footnotetext{
${ }^{*}$ Corresponding author. Tel: +1-214-768-1378; E-mail: xlgao@smu.edu.
} 


\section{Introduction}

Metamaterials are engineered materials with unusual properties deriving mainly from their micro-architecture rather than composition (e.g., Spadaccini, 2015; Chen et al., 2016). They have attracted increasing attention owing to their tailorable multi-functional properties that cannot be exhibited by naturally-occurring or traditionally-manufactured materials. Materials with a negative Poisson's ratio (PR) and materials with a non-positive coefficient of thermal expansion (CTE) are examples of mechanical metamaterials.

Poisson's ratio is positive for commonly used metallic materials. Novel cellular materials with negative PRs have been designed and fabricated in the last three decades (e.g., Lakes, 1987; Greaves et al., 2011; Gatt et al., 2013; Saxena et al., 2016; Huang and Chen, 2016). Such metamaterials, also known as auxetic materials (e.g., Prawoto, 2012; Saxena et al., 2016), expand transversally when stretched axially. Auxetic materials have been found to exhibit unique and enhanced mechanical and physical properties, which make them ideal for some important applications in aerospace and defense industries such as in sensors, deployable structures, body armor and combat helmets, and fasteners and rivets (e.g., Liu, 2006; Underhill, 2014).

In addition, conventional materials expand when heated up and shrink when cooled down, thereby displaying a positive CTE. Metamaterials with a zero or negative CTE have been proposed for applications in structures or devices subjected to temperature changes such as in space vehicles, naval ships, bridges, and pipelines (e.g., Yamamoto, et al., 2014; Chen et al., 2015; Wu et al., 2016; $\mathrm{Xu}$ and Pasini, 2016) to maintain thermal stability and reduce thermal stresses. The use of metallic cellular structures with a zero thermal expansion coefficient can mitigate thermomechanical stresses and extend service lives of such structures and devices. 
Metallic metamaterials with non-positive CTEs have been proposed using lattice structures. Lakes $(1996,2007)$ proposed a cellular structure with curved ribs composed of two dissimilar materials having different CTEs, which could achieve unbounded thermal expansion through material selections. Grima et al. (2007) constructed a 2-D lattice structure with its struts connected through hinges to form triangular units, in which the struts are designed to be made from dissimilar constituents. Their study showed that through adjusting geometrical parameters and material combinations, the CTE of the lattice structure can be tailored to be either positive or negative. Steeves et al. (2007) introduced a robust stretch-dominated bimaterial lattice that can have low thermal expansion but high stiffness. Miller et al. (2008) described a generalized mechanism for tailoring the CTE from a triangular sub-unit composed of corner-hinged beamlike elements. Through tessellations of this unit, many 2-D and 3-D structures could be designed to attain a tunable negative, zero or positive CTE. Palumbo et al. (2011) designed lattice structures with two constituent materials based on lightweight structures found in aerospace and spacecraft applications. These types of structures are similar to those proposed by Grima et al. (2007) that are fully triangular lattice structures made from more than two materials. Near-zero thermal expansion was achieved without incurring penalty in terms of stiffness and mass owing to the fully triangulated structure. Berger et al. (2011) modified the structure proposed in Steeves et al. (2007) by placing appropriately sized spacer between the unit cells for the bonded bimaterial structure. It was shown that such bonded lattices give thermomechanical responses that converge to those exhibited by pin-jointed lattice structure when the slenderness ratio of struts is small. Wei et al. (2016) designed six types of periodic planar lattices inspired by rotational symmetry in crystallography and presented an analytical solution for the effective CTE and biaxial stiffness of pin-jointed planar lattices. Hopkins et al. (2016) designed 2-D and 3-D 
microstructural architectures that can be tuned to achieve specific thermal expansion coefficients from positive to negative.

In these existing designs of metallic metamaterials, members of different materials are joined through pins, adhesive, welding or pressure-fit joints. This indicates that in each case two or more steps are involved in the fabrication process and manual work is required for assembling. Also, there are stress concentrations at the joints, which can lead to unexpected failures. Therefore, in order to fabricate metallic metamaterials that involve more cost-effective and reliable joints, new manufacturing methods are needed to mitigate these difficulties.

Studies have shown that there is no direct mechanistic relation between the mechanisms for a negative PR and those for a negative CTE (e.g., Sigmund and Torquato, 1996; Huang and Chen, 2016). At the molecular level, it was also shown that zeolite can possess a negative PR and a negative CTE simultaneously (Woodcock et al., 1999; Grima et al., 2000). These indicate that it is possible to have metamaterials that display both a negative CTE and a negative PR at the same time. One chiral lattice structure with bi-material ribs that can achieve a PR of -1 and negative thermal expansion has recently been designed and fabricated by Ha et al. (2015). However, the stiffness of the proposed chiral structure is compromised due to the bending and nodal rotation of the chiral structure. There is a lack of systematic studies on metamaterials exhibiting both negative PRs and non-positive CTEs. This further motivated the current work.

In this study, four planar bimaterial metallic lattice metamaterials are designed, which do not involve pins, adhesive, welding or pressure-fit joints and can be fabricated through laser-based additive manufacturing (e.g., Ding and Kovacevic, 2016). The effective Poisson's ratio and coefficient of thermal expansion of each designed metamaterial are evaluated. The effective Young's modulus is also computed to measure the load-bearing capability of each metamaterial. 
Three material combinations are considered, which include aluminum-Invar (Al-Invar), aluminum-stainless steel (Al-St) and stainless steel-Invar (St-Invar). Comprehensive parametric studies are performed, and structure-property relations are explored for each of the four designed metamaterials. Based on these studies, the metallic lattice structure for achieving both a negative PR and a non-positive CTE is identified, which can be fabricated through laser-based additive manufacturing.

\section{Newly Designed Metamaterials}

The metallic metamaterials designed in the current study are based on star-shaped re-entrant lattice structures. Re-entrant structures are known to be one class of auxetic structures that display negative Poisson's ratios (e.g., Lakes, 1987; Theocaris et al., 1997; Agnese and Scarpa, 2014; Gong et al., 2015; Saxena et al., 2016). To obtain a negative thermal expansion coefficient additionally, two types of struts made from two metallic materials with different CTEs are employed in the current design of each metamaterial. Combining the re-entrant star-shaped structure and the bi-material lattice design will lead to new metamaterials that can attain both a negative CTE and a negative PR, as demonstrated below.

Four metamaterials have been designed by following this combined approach. Figure 1 shows the four metamaterials designed, in each of which the red struts are made from the material with the higher CTE, while the blue struts are made from the material with the lower CTE. Each of the four designed metamaterials has a star-shaped, re-entrant, periodic lattice structure, whose unit cell is shown on its right. When heated, the thermal expansions of the struts are accommodated by their stretching and bending into the open spaces, resulting in a lower effective CTE. It can also be observed that each of the four metamaterials designs has a four-fold 
rotational symmetry that leads to an isotropic response under a homogeneous temperature change (e.g., Wei et al., 2016). In addition, all of the struts are assumed to be homogeneous, isotropic and linearly elastic and have a constant CTE, and the cross section for each of the struts is taken to have the same rectangular shape.

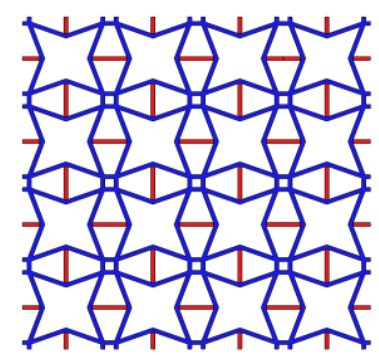

(a) Metamaterial \# 1

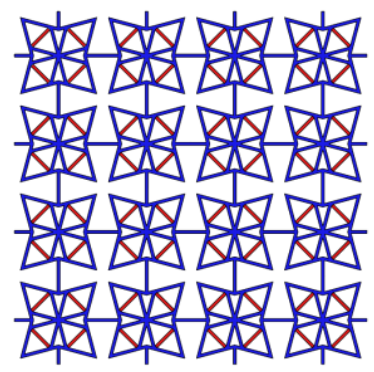

(c) Metamaterial \# 3
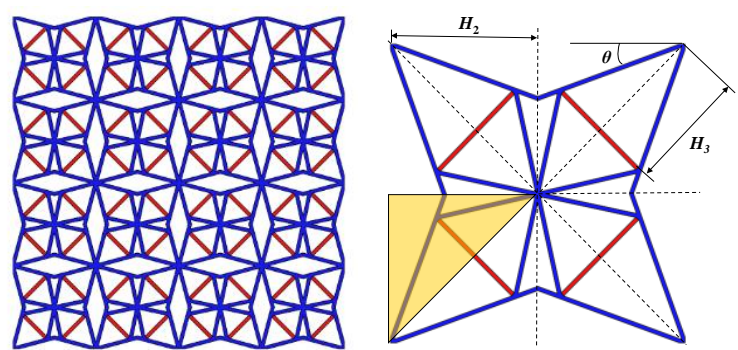

(b) Metamaterial \# 2
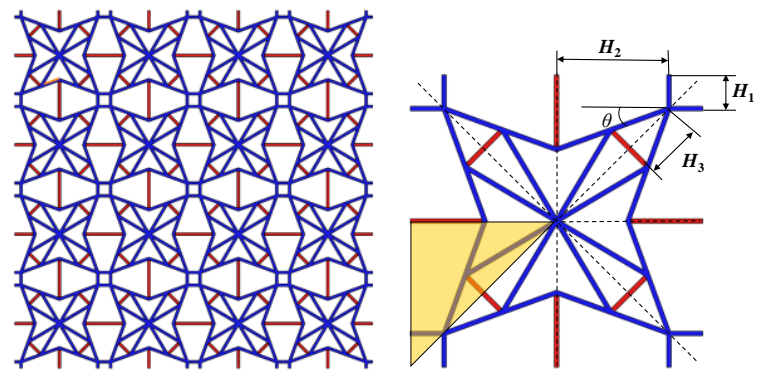

(d) Metamaterial \# 4

Fig. 1 Four metamaterials designed and their unit cells

It can be seen from the unit cells shown Figs. 1(a)-1(d) that the four designed metamaterials can be characterized in a unified manner by using three or four geometrical parameters: three parameters for metamaterials \# 1 and \# 2, including two length parameters $\left(H_{1}\right.$ and $H_{2}$ or $H_{2}$ and $H_{3}$ ) and one angle parameter $(\theta)$; four parameters for metamaterials \# 3 and \# 4, including three length parameters $\left(H_{1}, H_{2}\right.$ and $\left.H_{3}\right)$ and one angle parameter $(\theta)$. Note that the meaning of each parameter is kept the same for all of the four metamaterials to facilitate comparisons. In addition, in order to ensure the manufacturing feasibility of the four metamaterials, the following geometrical constraints have to be satisfied by the four parameters: 


$$
\begin{gathered}
H_{1}>0, H_{2}>0, \\
0<H_{3}<M, \quad M \equiv \frac{H_{2} \cos \left(45^{\circ}-\theta\right)}{\cos \theta}, \\
0 \leq \theta<45^{\circ} .
\end{gathered}
$$

In Eq. (2), $M$, as defined, is the maximum value that $H_{3}$ can take. Clearly, Eqs. (2) and (3) show that $M$ is a positive constant for each given value of $\theta$. By using $t$ and $M$, the following three non-dimensional parameters are introduced:

$$
p=\frac{H_{1}}{t}, q=\frac{H_{2}}{t}, m=\frac{H_{3}}{M} .
$$

These three parameters will be used in plotting the numerical results in Section 4.

In addition, the unit cell for each metamaterial can be divided into eight sectors (see one sector indicated by a yellow shaded area for each case shown in Fig. 1). Through adjusting the sector (and thus the unit cell) geometry, the anisotropy of the metamaterial can be tuned to satisfy the needs for specific applications. In particular, by making the eight sectors identical, isotropic in-plane material properties can be obtained. In the current study, only isotropic cases with identical sectors are considered, as indicated in Fig. 1.

Three materials, i.e., aluminum (Al) 7075, stainless steel (St) 431 and Invar, are chosen for the struts, and three material combinations, i.e., Al-Invar, Al-St and St-Invar, are considered in the current study. The values of Young's modulus and CTE for the strut materials are listed in Table 1, which are adopted from Wei et al. (2016). In addition, the Poisson's ratio and density are assumed to remain constant over the temperature range considered for each strut material, as indicated in Table 1. The values of $v$ and $\rho$ for the strut materials at the room temperature $T=$ $20^{\circ} \mathrm{C}$ listed in Table 1 are commonly used ones (e.g., www.efunda.com; Corredor et al., 2013). 
Table 1 Material properties for Al, St and Invar struts

\begin{tabular}{|c|c|c|c|c|c|}
\hline \multirow{2}{*}{ Material } & $\begin{array}{c}\text { Young's modulus } \\
E(\mathrm{GPa})\end{array}$ & $\begin{array}{c}\text { Coefficient of thermal expansion } \\
\alpha\left(\mathrm{ppm} /{ }^{\circ} \mathrm{C}\right)\end{array}$ & $\begin{array}{c}\text { Poisson's ratio } \\
v\end{array}$ & $\begin{array}{c}\text { Density } \\
\rho\left(\mathrm{kg} / \mathrm{m}^{3}\right)\end{array}$ & $\begin{array}{c}\text { Temperature } \\
T\left({ }^{\circ} \mathrm{C}\right)\end{array}$ \\
\hline \multirow{2}{*}{$\mathrm{Al} 7075$} & 71 & 23.0 & 0.33 & 2810 & 20 \\
\cline { 2 - 6 } & 66 & 24.3 & 0.33 & 2810 & 200 \\
\hline \multirow{2}{*}{$\mathrm{St} 431$} & 193 & 10.3 & 0.28 & 7800 & 20 \\
\cline { 2 - 6 } & 178 & 10.3 & 0.28 & 7800 & 200 \\
\hline \multirow{2}{*}{ Invar } & 144 & 1.1 & 0.29 & 8050 & 20 \\
\cline { 2 - 6 } & 135 & 2.5 & 0.29 & 8050 & 200 \\
\hline
\end{tabular}

The formula for the relative density $\rho_{r}$, defined as $\rho_{r}=\rho / \rho_{H}$ where $\rho_{H}$ is the strut material with the higher CTE, for each of the four designed metamaterials is given in Table 2, which is derived directly from each unit cell shown in Fig. 2.

Table 2 Relative density for the four designed metamaterials

\begin{tabular}{|c|l|}
\hline Metamaterial & Relative Density \\
\hline$\# 1$ & $\rho_{r}=\frac{\left(4 \rho_{H} H_{1}+4 \rho_{H} H_{2} \tan \theta+8 \rho_{L} H_{1}+8 \rho_{L} H_{2} / \cos \theta\right) t}{4 \rho_{H}\left(H_{1}+H_{2}\right)^{2}}$ \\
\hline$\# 2$ & $\rho_{r}=\frac{\left(8 \rho_{H} H_{3} \tan \theta_{1}+8 \rho_{L} H_{2} / \cos \theta+8 \rho_{L} H_{3} \tan \theta_{1} / \sin \theta_{2}\right) t}{4 \rho_{H} H_{2}^{2}}$ \\
\hline$\# 3$ & $\rho_{r}=\frac{\left(8 \rho_{H} H_{3} \tan \theta_{1}+4 \rho_{L} H_{1}+4 \rho_{L} H_{2} \tan \theta+8 \rho_{L} H_{2} / \cos \theta+8 \rho_{L} H_{3} \tan \theta_{1} / \sin \theta_{2}\right) t}{4 \rho_{H}\left(H_{1}+H_{2}\right)^{2}}$ \\
\hline 44 & $\rho_{r}=\frac{\left(4 \rho_{H} H_{1}+4 \rho_{H} H_{2} \tan \theta+8 \rho_{H} H_{3} \tan \theta_{1}+8 \rho_{L} H_{1}+8 \rho_{L} H_{2} / \cos \theta+8 \rho_{L} H_{3} \tan \theta_{1} / \sin \theta_{2}\right) t}{4 \rho_{H}\left(H_{1}+H_{2}\right)^{2}}$ \\
\hline
\end{tabular}

Here $\theta_{1}=45^{\circ}-\theta, \theta_{2}=\tan ^{-1}\left[H_{3} \tan \theta_{1} /\left(\sqrt{2} H_{2}-H_{3}\right)\right], t$ is the thickness of the struts, and $\rho_{L}$ and $\rho_{H}$ are the densities of the strut materials with the lower CTE and higher CTE, respectively.

\section{Finite Element Simulations}

\subsection{Periodic boundary conditions}

For the four designed metamaterials shown in Figs. 1(a)-1(d), which are periodic lattice structures, the simulations are carried out by employing the unit cells identified, and the periodic boundary conditions are enforced. The use of periodic boundary conditions ensures the continuity of displacements and rotations between adjacent cells (e.g., Li et al., 2005, 2006). 
To illustrate the periodic boundary conditions for the current models, metamaterial \# 1 (see Fig. 1(a)) is considered here as an example. The lattice structure of this metamaterial is periodic, i.e., each node on one side (e.g., $A^{+}$) of the unit cell has a matched node on the opposite side of the unit cell (e.g., $A^{-}$), as shown in Fig. 2.

For a uniaxially loaded specimen in the $i$ th direction subjected to a prescribed strain $\varepsilon_{i}$, the periodic boundary conditions can be written as (e.g., Li et al., 2005, 2006)

$$
u_{i}^{k^{+}}-u_{i}^{k^{-}}=\varepsilon_{i}\left(x_{i}^{k^{+}}-x_{i}^{k^{-}}\right), \quad i \in\{1,2\} ; \varphi^{k^{+}}-\varphi^{k^{-}}=0
$$

where $x_{i}^{k^{+}}$and $x_{i}^{k^{-}}$are, respectively, the positions of the matched nodes $k^{+}$and $k^{-}$on the specimen boundary lines with outward-pointing unit normal vectors $\mathbf{e}_{i}$ and $-\mathbf{e}_{i}, u_{i}^{k^{+}}$and $u_{i}^{k^{-}}$are, respectively, the normal displacement components of $k^{+}$and $k^{-}$, and $\varphi^{k+} \varphi^{k-}$ are, respectively, the rotations of $k^{+}$and $k^{-}$.

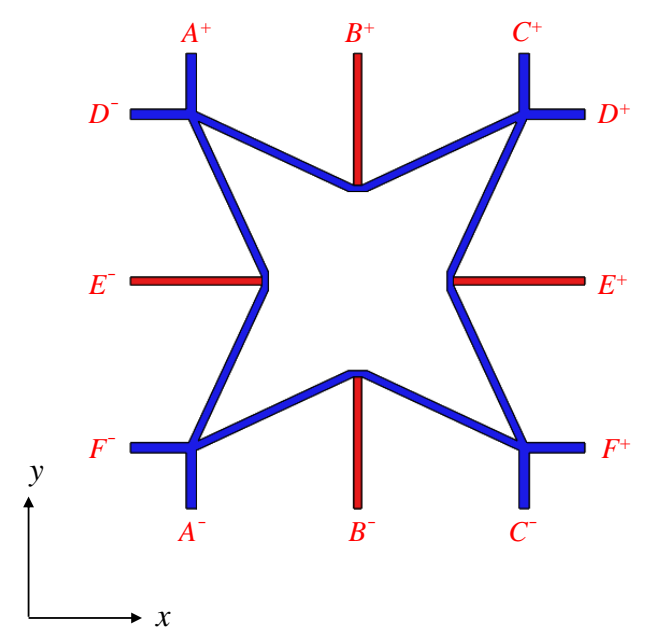

Fig. 2 Matched nodes on the boundary of the unit cell for metamaterial \# 1

For the unit cell of metamaterial \# 1 shown in Fig. 2 under the uniaxial loading in the $y$ direction with a prescribed strain $\varepsilon_{a}$, the use of Eq. (5) gives the periodic boundary conditions as 


$$
\begin{aligned}
& u_{x}^{A^{+}}=u_{x}^{A^{-}}, \quad u_{x}^{B^{+}}=u_{x}^{B^{-}}, \quad u_{x}^{C^{+}}=u_{x}^{C^{-}}, \\
& u_{y}^{A^{+}}-u_{y}^{A^{-}}=u_{y}^{B^{+}}-u_{y}^{B^{-}}=u_{y}^{C^{+}}-u_{y}^{C^{-}}, \\
& u_{x}^{D^{+}}-u_{x}^{D^{-}}=u_{x}^{E^{+}}-u_{x}^{E^{-}}=u_{x}^{F^{+}}-u_{x}^{F^{-}}, \\
& u_{y}^{D^{+}}=u_{y}^{D^{-}}, \quad u_{y}^{E^{+}}=u_{y}^{E^{-}}, \quad u_{y}^{F^{+}}=u_{y}^{F^{-}}, \\
& \varphi^{A^{+}}=\varphi^{A^{-}}, \quad \varphi^{B^{+}}=\varphi^{B^{-}}, \quad \varphi^{C^{+}}=\varphi^{C^{-}}, \\
& \varphi^{D^{+}}=\varphi^{D^{-}}, \quad \varphi^{E^{+}}=\varphi^{E^{-}}, \quad \varphi^{F^{+}}=\varphi^{F^{-}},
\end{aligned}
$$

where $A^{+}, A^{-} ; B^{+}, B^{-} ; C^{+}, C^{-} ; D^{+}, D^{-} ; E^{+}, E^{-}$; and $F^{+}, F^{-}$are six pairs of the matched nodes on the

boundary, as displayed in Fig. 2. To implement these periodic boundary conditions in the finite element simulations, the matched nodes of $A^{+}, A$ - and $D^{+}, D$ - are taken to be the reference nodes. To prevent any rigid body motion, the node $A^{-}$is fixed in each simulation. These periodic boundary conditions are similar to those presented in Harders et al. (2005) without derivations. For the uniaxial deformation in the $x$-direction, the periodic boundary conditions can be similarly obtained from the general formulas listed in Eq. (5).

ANSYS Mechanical APDL 16.2 (ANSYS Inc., 2015) is employed to perform all the FE simulations, in which the periodic boundary conditions including those listed in Eq. (6) are implemented through constraint equations.

\subsection{Finite element mesh}

In order to simulate the planar lattice metamaterials designed, several element types available in the element library of ANSYS can be chosen. The 3-D beam and shell elements have advantages over 3-D solid elements in computation efficiency. Also, for the struts in the lattice structures considered in the current study, one dimension is larger than the other two dimensions. Hence, beam elements are more suitable. As a result, the element BEAM 189 in ANSYS is selected. This beam element is based on the Timoshenko beam theory (e.g., Ma et al., 2008; Roque et al., 2013; Gao, 2015) and is a quadratic three-node beam element suitable for analyzing 
slender to moderately thick beam structures. This element has six degrees of freedom at each node: translation in each of the three directions and rotation about each of the three directions.

For illustration purposes, only metamaterial \# 1 and the material combination Al-Invar are considered in the finite element simulations presented in this section.

Figure 3 shows the finite element mesh of the unit cell for metamaterial \# 1, which is constructed using the BEAM 189 elements (totaling 940 for the unit cell with $H_{1}=10 \mathrm{~mm}, H_{2}=$ $30 \mathrm{~mm}, \theta=30^{\circ}$, and $t=w=2 \mathrm{~mm}$ displayed here). Based on a convergence study, a size of 0.5 $\mathrm{mm}$ in the axial length is chosen for each BEAM 189 element. The cross-sectional dimensions of the beam element are those of the strut defined by $t \times w$, where $t$ is the strut thickness and $w$ is the strut width (i.e., the out-of-plane length in the $z$ direction). Note that in Fig. 3 and in Fig. 6 the beam elements are displayed with nodes (including corner nodes) on cross-section boundaries to better visualize the finite element mesh, even though each BEAM 189 element can be represented using a one-dimensional line element along the axial direction because of the uniform cross-section adopted in constructing the beam element (ANSYS, 2015). In addition, it should be mentioned that no out-of-plane deformation is considered in the current study.

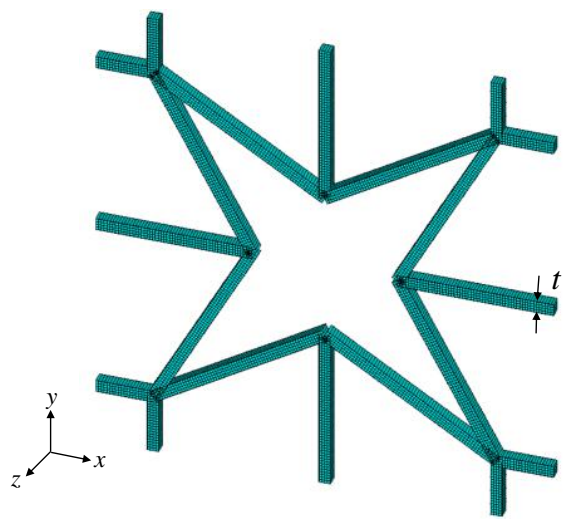

Fig. 3 Finite element mesh of the unit cell for metamaterial \# 1

\subsection{Simulation results}

In addition to the effective PR and CTE, the effective Young's modulus is computed as a 
measure of the structural load-carrying capacity. The temperature change and mechanical loading are applied independently. Thermal deformations are considered for the temperature change from the room temperature $\left(20{ }^{\circ} \mathrm{C}\right)$ to $180{ }^{\circ} \mathrm{C}$. The effective CTE is calculated by measuring the length change between the matched nodes on two sides of the unit cell. The effective Young's modulus and PR are determined from the unit cell (see Fig. 2) under the periodic boundary conditions by applying a uniform displacement on the top side of the unit cell in the $y$ direction and by setting the $y$ displacement on the bottom side to be zero.

The effective Poisson's ratio $v$, the effective Young's modulus $E$ and the effective coefficient of thermal expansion $\alpha$ can be obtained from Eqs. (7)-(9):

$$
\begin{gathered}
v=-\frac{\varepsilon_{x}}{\varepsilon_{a}}, \\
E=\frac{F_{R}}{A_{e f f} \varepsilon_{y}}, \\
\alpha=\frac{\varepsilon_{y}^{T}}{\Delta T},
\end{gathered}
$$

where $\varepsilon_{a}$ is the applied strain in the $y$-direction, $\varepsilon_{x}$ is the lateral strain in the $x$-direction that is induced by $\varepsilon_{a}$ and can be computed from the nodal displacements at the nodes $D^{+}$and $D^{-}$ (obtainable from the output data of the finite element simulation) according to

$$
\varepsilon_{x}=\frac{u_{x}^{D^{+}}-u_{x}^{D^{-}}}{2\left(H_{1}+H_{2}\right)}
$$

$F_{R}$ is the resultant force in the $y$-direction that can be determined from the summation of the nodal forces at the nodes $A^{+}, B^{+}$and $C^{+}$on the top side of the unit cell, $A_{\text {eff }}\left[=2\left(H_{1}+H_{2}\right) \times w\right]$ is the effective area perpendicular to the loading direction (e.g., Li et al., 2003, 2005a), $\Delta T$ is the temperature change, and $\varepsilon_{y}^{T}$ is the thermal strain in the $y$-direction that is induced by the temperature change and can be computed from the nodal displacements at the nodes $A^{+}$and $A^{-}$ 
due to the temperature change (i.e., $\left(u_{x}^{A^{+}}\right)^{T}$ and $\left.\left(u_{x}^{A^{-}}\right)^{T}\right)$ according to

$$
\varepsilon_{y}^{T}=\frac{\left(u_{x}^{A^{+}}\right)^{T}-\left(u_{x}^{A^{-}}\right)^{T}}{2\left(H_{1}+H_{2}\right)} .
$$

Figure 4 displays the effective Poisson's ratio $v$ and the normalized effective CTE $\alpha^{*}$ (三 $\alpha / \alpha_{A l} ; \alpha_{A l}=23.0 \mathrm{ppm} /{ }^{\circ} \mathrm{C}$ ) varying with the strut thickness $t$ based on the unit cell shown in Fig. 1(a) with the given parameters $H_{1}=10 \mathrm{~mm}, H_{2}=100 \mathrm{~mm}$ and $\theta=20^{\circ}$. The values of $v$ and $\alpha^{*}$ are obtained from Eqs. (7) and (9). It is seen from Fig. 4 that both $v$ and $\alpha^{*}$ increase with the increase of $t$, which means that the effective PR and CTE become less negative when the strut thickness gets larger. The variation in the strut thickness $t$ from $1 \mathrm{~mm}$ to $5 \mathrm{~mm}$ leads to an increase of $5.5 \%$ for the effective PR $v$ and an increase of $2.94 \%$ for the effective CTE $\alpha^{*}$.

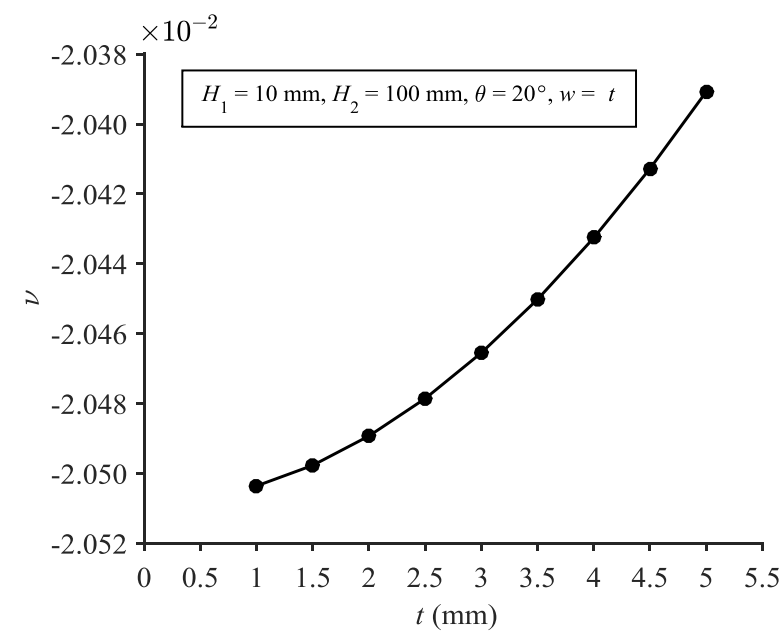

(a)

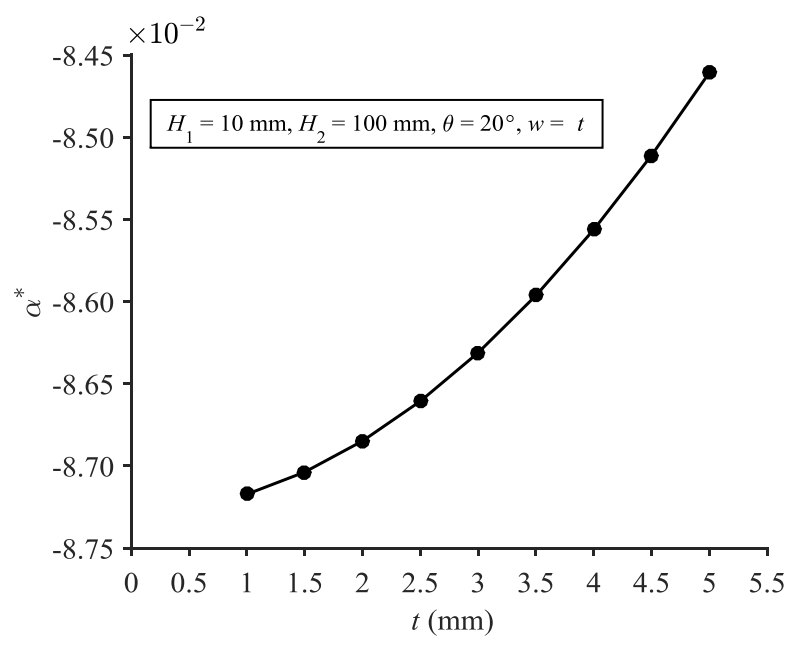

(b)

Fig. 4 Variations of (a) the effective Poisson's ratio $v$ and (b) the normalized effective CTE $\alpha^{*}$ (三 $\left.\alpha / \alpha_{A l}\right)$ with the strut thickness $t$ for metamaterial \# 1 with $H_{1}=10 \mathrm{~mm}, H_{2}=100 \mathrm{~mm}, \theta=20^{\circ}$ and the Al-Invar combination

The variations of the normalized effective Young's modulus $E^{*}\left(\equiv E / E_{A l} ; E_{A l}=71 \mathrm{GPa}\right)$ and the relative density $\rho_{r}$ with the strut thickness $t$ are shown in Fig. 5 based on the same unit cell with $H_{1}=10 \mathrm{~mm}, H_{2}=100 \mathrm{~mm}$ and $\theta=20^{\circ}$. The values of $E^{*}$ are obtained from Eq. (8), while those of $\rho_{r}$ are computed using the formula given in Table 2 for metamaterial \# 1 that estimates 
the relative density without considering the overlapping near the strut joints.

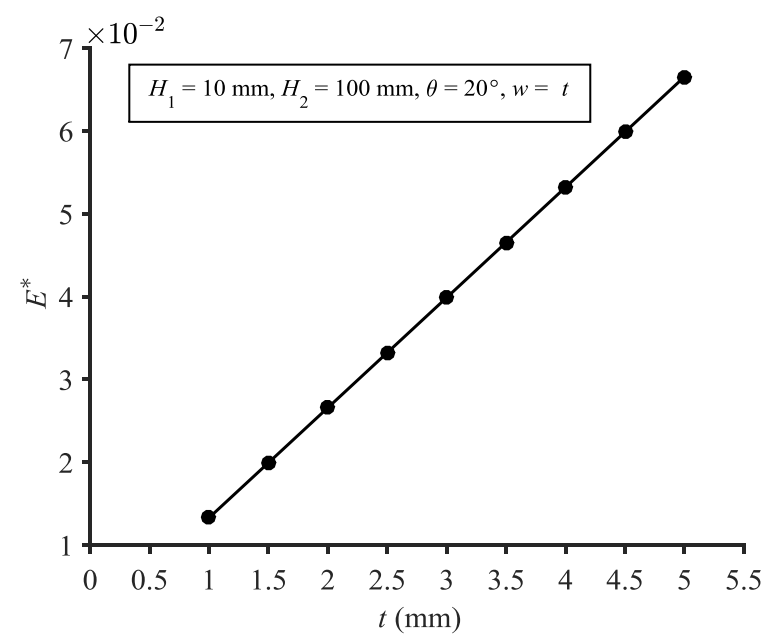

(a)

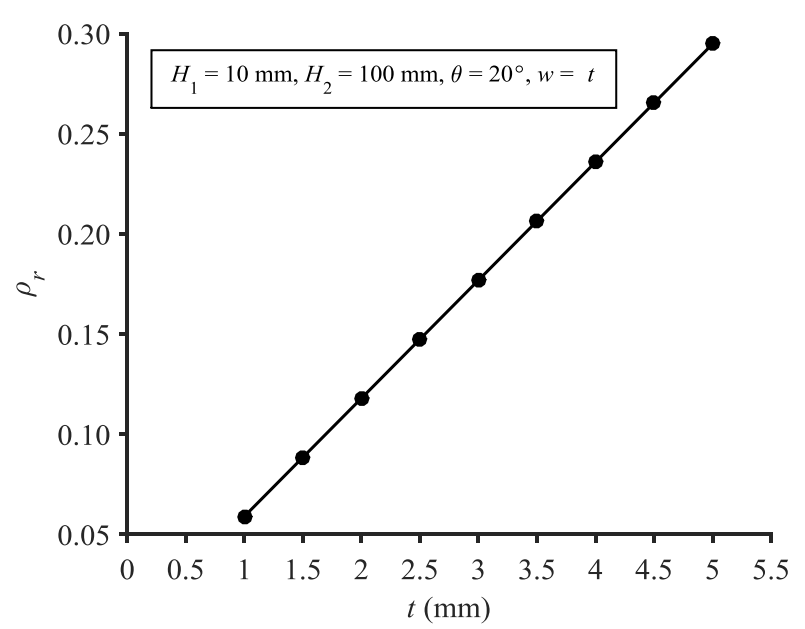

(b)

Fig. 5 Variation of (a) the normalized effective Young's modulus $E^{*}\left(\equiv E / E_{A l}\right)$ and (b) the relative density $\rho_{r}$ with the strut thickness $t$ for metamaterial \# 1 with $H_{1}=10 \mathrm{~mm}, H_{2}=100 \mathrm{~mm}$, $\theta=20^{\circ}$ and the Al-Invar combination

It is observed from Fig. 5 that both $E^{*}$ and $\rho_{r}$ increase with the increase of $t$. According to the scaling law for cellular materials given by $E^{*}=a\left(\rho_{r}\right)^{b}$ (e.g., Gibson and Ashby, 1997; Ashby, 2006), the deformation mode for the cellular material is stretch-dominated when $b$ is close to one and is bending-dominated when $b$ is around 2. Through fitting $E^{*}$ with $\rho_{r}$, it is found that $a=$ 22.6, $b=1.001$, which indicates that the deformation mode for metamaterial \# 1 is stretchdominated. This agrees with what is predicted by Maxwell's stability criterion (Ashby, 2006), which gives $M=b-2 j+3=7$, with the number of struts $b$ being 20 and the number of joints $j$ being 8 in the unit cell, for matematerial \# 1, which can be modeled as a pin-joined 2-D frame. With $M=7>0$, deformations of matematerial \# 1 should be stretch-dominated according to Maxwell's stability criterion.

Figure 6 shows the simulated deformations of the unit cell for metamaterial \# 1 induced by a uniaxial compression in the $y$ direction and a temperature increase of $160{ }^{\circ} \mathrm{C}$, respectively. From Fig. 6(a), it is clearly seen that the metamaterial contracts in the $x$ direction under the uniaxial compression in the $y$ direction. This can be attributed to the well-known folding and unfolding 
mechanisms exhibited by re-entrant structures under compressive and tensile loads, respectively (e.g., Lakes, 1987). Also, it is observed from Fig. 6(b) that the metamaterial undergoes a thermal contraction upon the uniform temperature increase. This results from the use of two strut materials with different values of CTE and the expansions of these struts into free spaces available, which is known to be one mechanism for achieving a negative CTE (e.g., Grima et al., 2007). These demonstrate that the bi-material planar lattice structure of metamaterial \# 1 designed in the current study can indeed exhibit a negative PR and a negative CTE simultaneously.

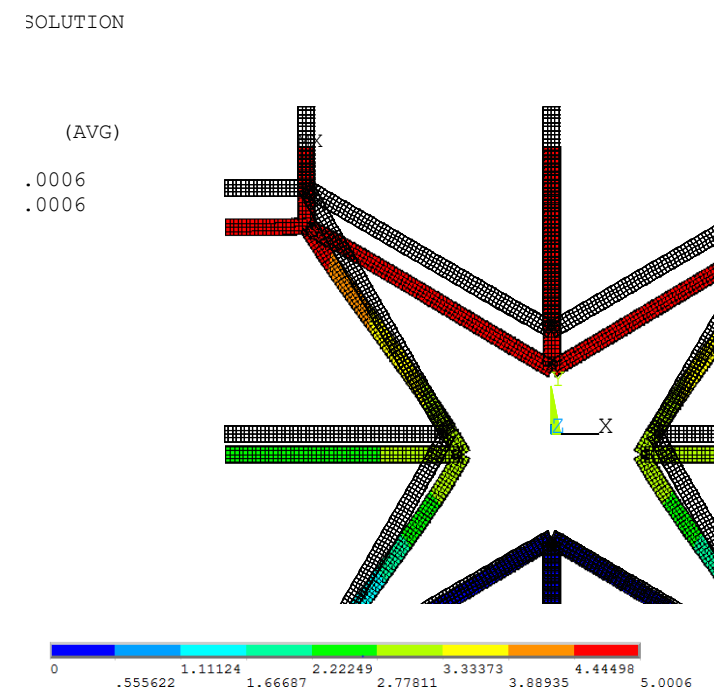

(a)

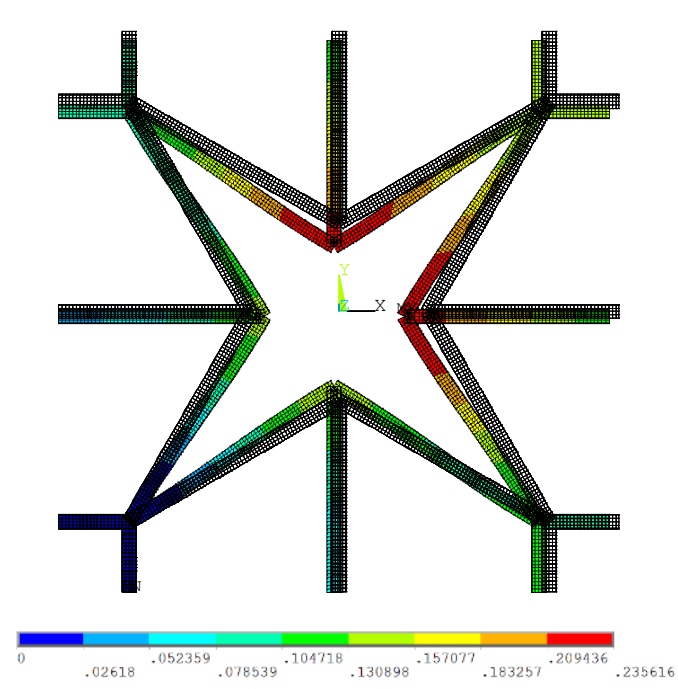

(b)

Fig. 6 Deformation of the unit cell for metamaterial \# 1 with $H_{1}=10 \mathrm{~mm}, H_{2}=30 \mathrm{~mm}, \theta=30^{\circ}, t$ $=w=2 \mathrm{~mm}$ and the Al-Invar combination induced by (a) a uniaxial compression in the $y$ direction; (b) a temperature increase of $160^{\circ} \mathrm{C}$. The black lines stand for the undeformed configurations, and the color lines denote the deformed configurations. Also, the colored legend bars represent displacements, with the unit in millimeters for each case.

\section{Parametric Study}

To understand how geometrical parameters and material combinations influence the properties of each metamaterial designed in Section 2, parametric studies have been conducted 
by using the unit cell-based finite element models described in Section 3. The numerical results are presented and discussed in this section.

\subsection{Metamaterial \# 1}

For this metamaterial, the unit cell is displayed in Fig. 1(a), and the finite element simulation results showing the effects of the strut thickness on the effective Poisson's ratio, CTE, Young's modulus, and the relative density have been presented in Section 3.

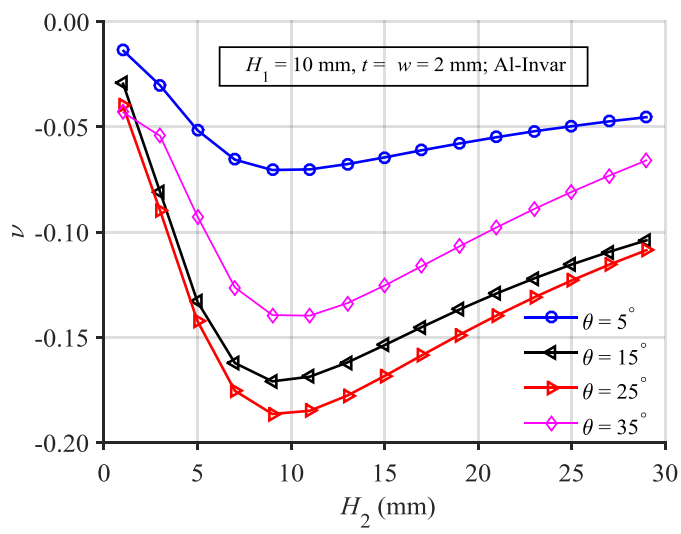

(a)

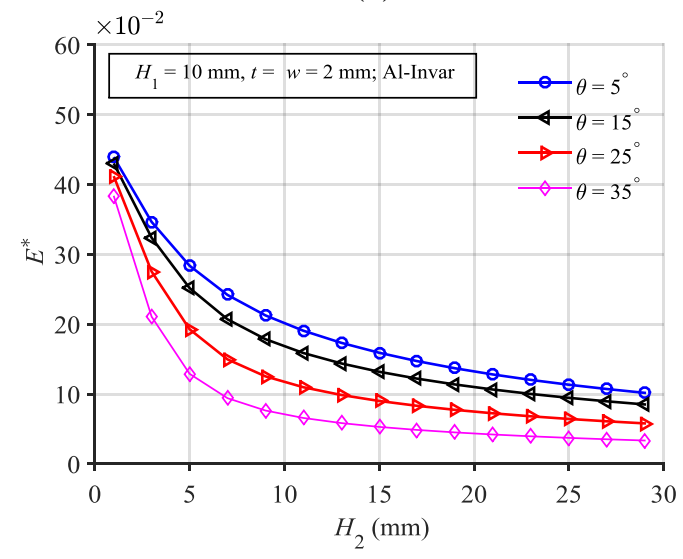

(c)

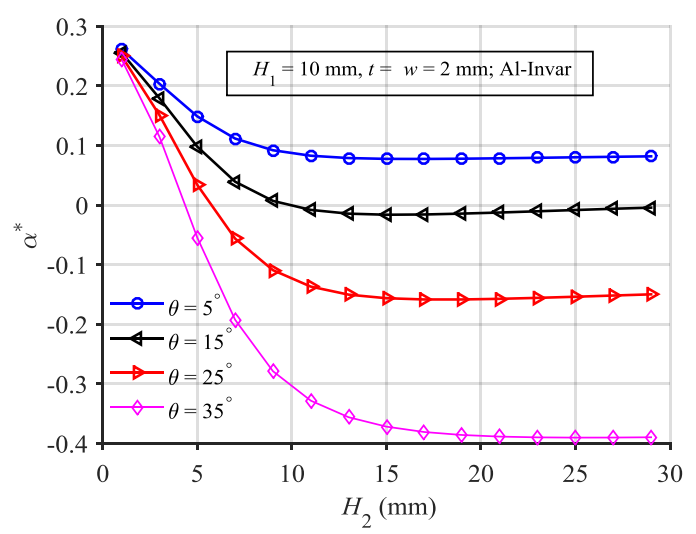

(b)

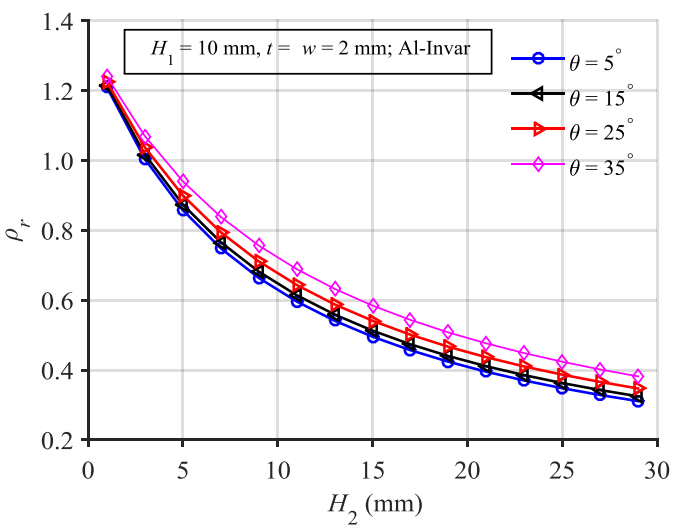

(d)

Fig. 7 Variations of the effective properties of metamaterial \# 1 with $H_{2}$ and $\theta$ : (a) the effective Poisson's ratio $v$, (b) the normalized effective CTE $\alpha^{*}\left(\equiv \alpha / \alpha_{A l}\right)$, (c) the normalized effective Young's modulus $E^{*}\left(\equiv E / E_{A l}\right)$, and (d) the relative density $\rho_{r}$

Figure 7 shows how the effective Poisson's ratio $v$, CTE $\alpha^{*}$, Young's modulus $E^{*}$, and the relative density $\rho_{r}$ vary with $H_{2}$ and $\theta$. The numerical values of $v, \alpha^{*}$ and $E^{*}$ are obtained from 
Eqs. (7)-(9) after each finite element simulation is completed. The values for $\rho_{r}$ displayed in Fig. 7(d) are computed using the formula for metamaterial \# 1 listed in Table 2.

Figure 7(a) displays the effective Poisson's ratio $v$ varying with $H_{2}$ and $\theta$. It is clearly seen that for each value of $\theta, v$ is negative over the entire range of $H_{2}$, and $v$ reaches a maximum value (in magnitude) near $H_{2} / H_{1}=1$. After this extremal point, $v$ increases with $H_{2}$ for each value of $\theta$ under consideration. From Fig. 7(b), it is seen that the normalized effective CTE $\alpha^{*}$ first decreases and then remains almost unchanged with the increase of $H_{2}$ for all four lattice structures considered. The transition point for each of the lattice structures with a different value of the re-entrant angle $\theta$ occurs after $H_{2}$ becomes larger than $H_{1}$. This means that when the ratio of $H_{2} / H_{1}<1$, a change in $H_{2}$ leads to a considerably larger change in $\alpha^{*}$ than that when $H_{2} / H_{1}>$ 1 in each of the four cases considered. In addition, it is observed that $\theta$ has a larger effect on $\alpha^{*}$ than $H_{2}$ after $H_{2} / H_{1}$ becomes greater than 1 . With the increase of $\theta, \alpha^{*}$ changes dramatically from positive values to negative ones. This implies that a larger re-entrant angle $\theta$ is more beneficial in attaining a more negative CTE.

Figures 7(c) and 7(d) show, respectively, the normalized effective Young's modulus $E^{*}$ and the relative density $\rho_{r}$ changing with $H_{2}$ for four different values of $\theta$. It is seen that both $E^{*}$ and $\rho_{r}$ decrease with $H_{2}$. However, they exhibit different variation trends as $\theta$ changes: $E^{*}$ decreases with $\theta$, while $\rho_{r}$ increases with $\theta$. This can be attributed to the fact that a larger re-entrant angle brings the entire lattice structure more inward that reduces the void area and thus increases the relative density. On the other hand, a larger re-entrant angle makes the lattice structure more compliant, thereby leading to a lower stiffness (Young's modulus). This differs from the general trend that the stiffness of a regular cellular material increases with the relative density (e.g., Gibson and Ashby, 1997; Li et al., 2003). In summary, in order to obtain the largest negative PR 
and CTE (in magnitude), the ratio of $H_{2} / H_{1}$ should be chosen to be close to one, and an appropriate value of $\theta$ should be used to obtain the desired values for the effective PR and CTE without significantly compromising stiffness.

Figure 8 depicts the variations of the properties of metamaterial \# 1 for three different material combinations. Figure 8(a) displays how the effective Poisson's ratio $v$ changes with $\theta$ for the three material combinations. For each material combination, $v$ reaches a largest negative value (in magnitude) when $\theta$ changes from $0^{\circ}$ to $40^{\circ}$ : at $\theta=10^{\circ}$ for the Al-Invar combination, at $\theta=20^{\circ}$ for the St-Invar combination and at $\theta=15^{\circ}$ for the Al-St combination. It is also clear from Fig. 8(a) that $v$ does not decrease monotonically with the increase of $\theta$. The Al-St combination gives the maximum negative Poisson's ratio of -0.1529 , and the Al-Invar and StInvar combinations yield the maximum negative Poisson's ratios of -0.0314 and -0.0478 , respectively. From Fig. 8(b), it is seen that the metamaterial with the Al-St combination exhibits a positive CTE with the increase of $\theta$, while those with the Al-Invar and St-Invar combinations show a CTE decreasing from $\alpha^{*}=0.1087$ to -0.5692 and from $\alpha^{*}=0.1088$ to -0.1340 , respectively. The transition point from positive to negative values occurs around $\theta=17^{\circ}$ for the Al-Invar combination and around $\theta=28^{\circ}$ for the St-Invar combination. The different CTE ratio for each material combination (see Table 1) is a reason why different responses to the change of $\theta$ are obtained.

From Fig. 8(c), it is seen that $E^{*}$ decreases with the increase of $\theta$ for all the three material combinations. Also, it is found that the metamaterial with the Al-Invar combination has the lowest value of $E^{*}$ among the three material combinations. For the metamaterial with the Al-St combination, $E^{*}$ exhibits the largest change among the three when $\theta$ goes from $0^{\circ}$ to $40^{\circ}$. Figure 8(d) shows that the relative density $\rho_{r}$ is the largest for the metamaterial with the Al-Invar 
combination and the smallest for that with the St-Invar combination. In addition, it is observed that $\rho_{r}$ increases as $\theta$ increases for each of the three material combinations considered.

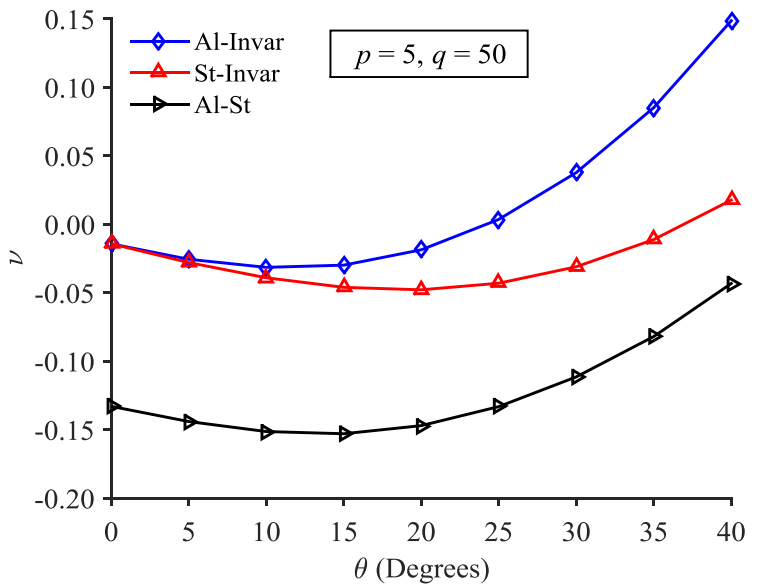

(a)

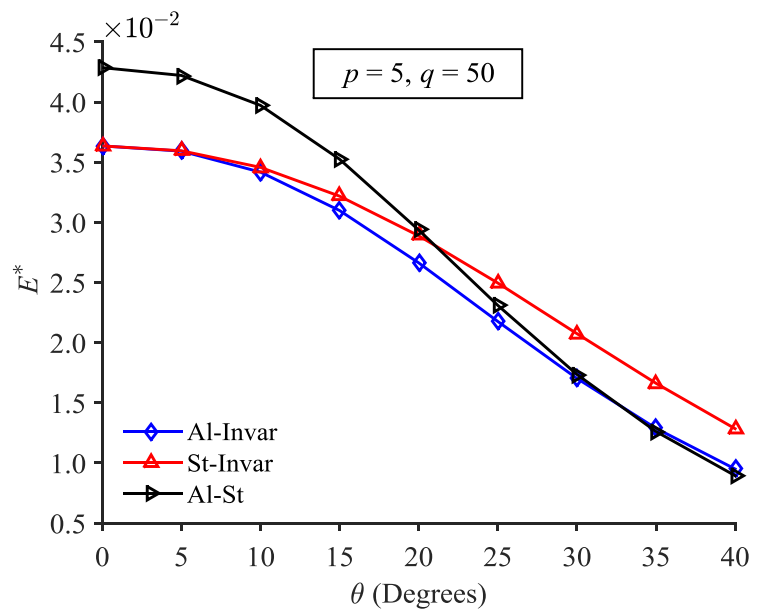

(c)

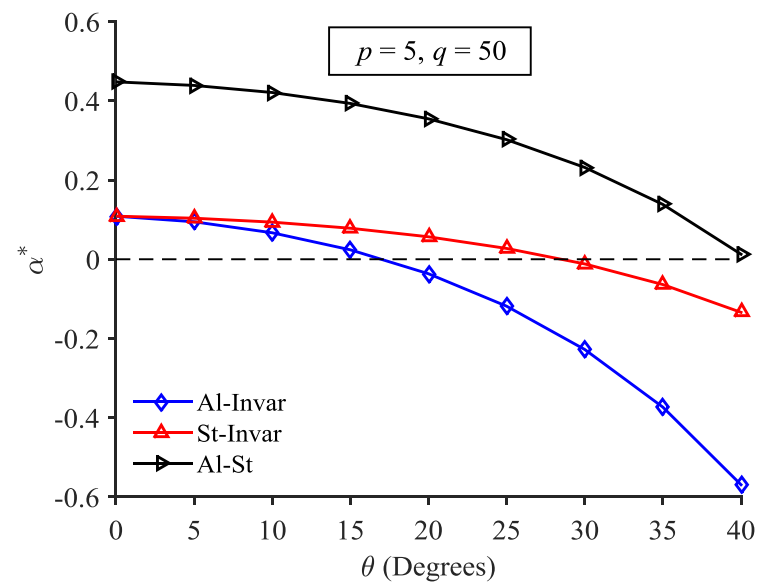

(b)

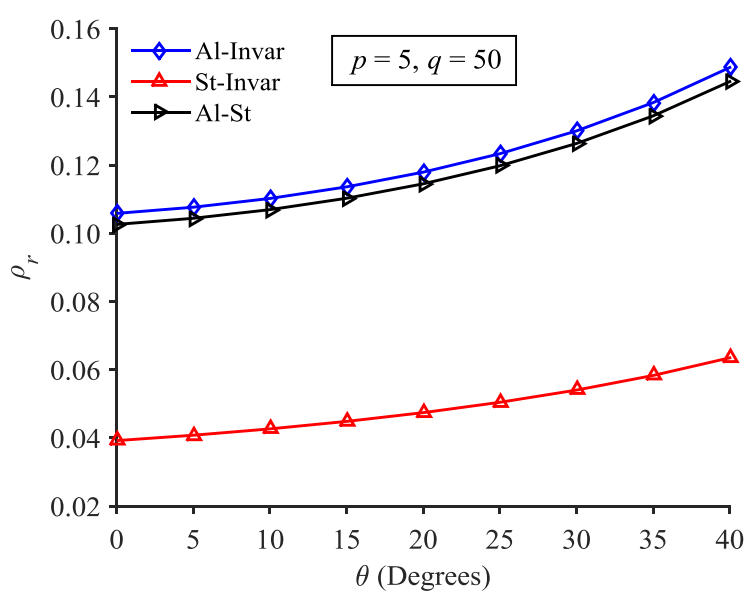

(d)

Fig. 8 Variations of the properties of metamaterial \# 1 with $\theta$ for three material combinations: (a) the effective Poisson's ratio $v,(\mathrm{~b})$ the normalized effective CTE $\alpha^{*}$ ( $\equiv \alpha / \alpha_{A l}$ ), (c) the normalized effective Young's modulus $E^{*}\left(\equiv E / E_{A l}\right)$, and (d) the relative density $\rho_{r}$. The cross-section of each strut is square with $t=w=2 \mathrm{~mm}$.

\subsection{Metamaterial \# 2}

For this metamaterial, the unit cell is displayed in Fig. 1(b). From Figs. 1(a) and 1(b), it can be seen that the main difference between metamaterial \# 2 and metamaterial \# 1 is that there exists an interior structure within the basic star-shaped structure for metamaterial \# 2. Also, $H_{3}$ is a new parameter whose effects on the metamaterial properties need to be studied along with those of $\mathrm{H}_{2}$ 
and $\theta$.

Figure 9 shows the variations of the effective Poisson's ratio $v$, CTE $\alpha^{*}$, Young's modulus $E^{*}$, and the relative density $\rho_{r}$ with the parameter $H_{3}$ and the strut thickness $t$. The numerical values of $v, \alpha^{*}$ and $E^{*}$ are obtained from Eqs. (7)-(9) after each finite element simulation based on the unit cell shown in Fig. 1(b) is completed. The values for $\rho_{r}$ are computed using the formula for metamaterial \# 2 given in Table 2.

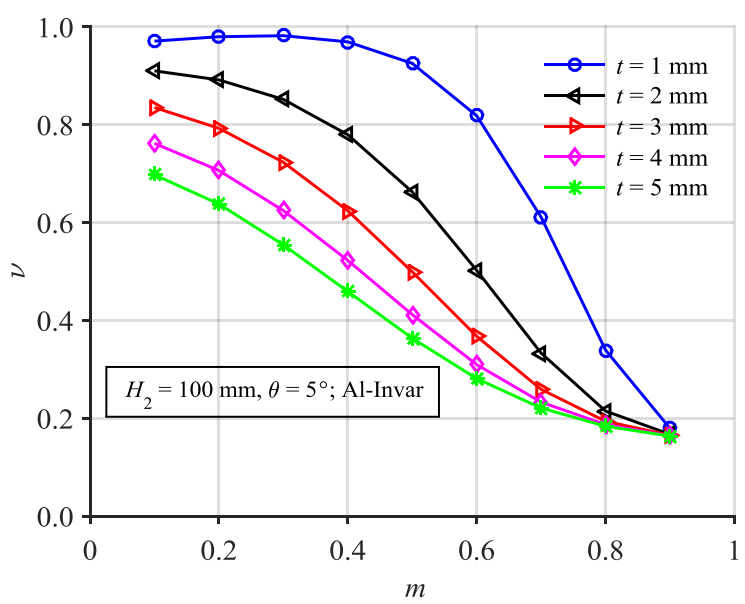

(a)

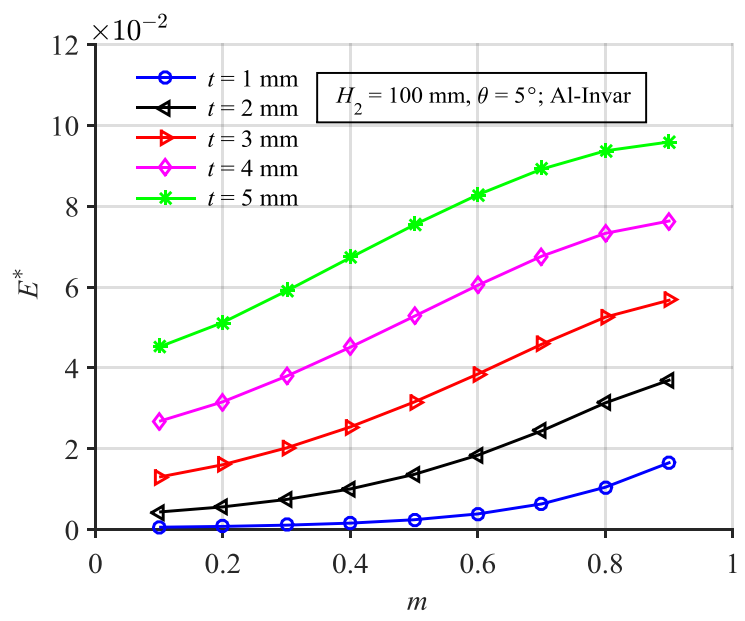

(c)

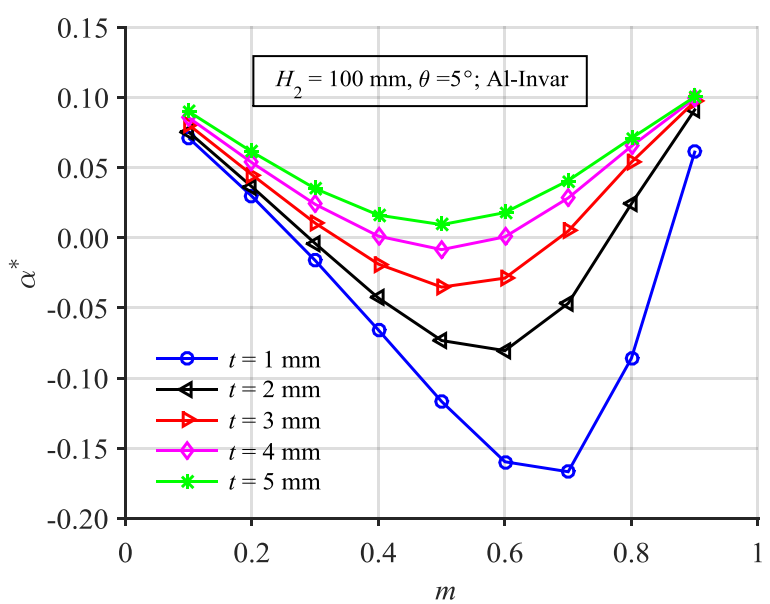

(b)

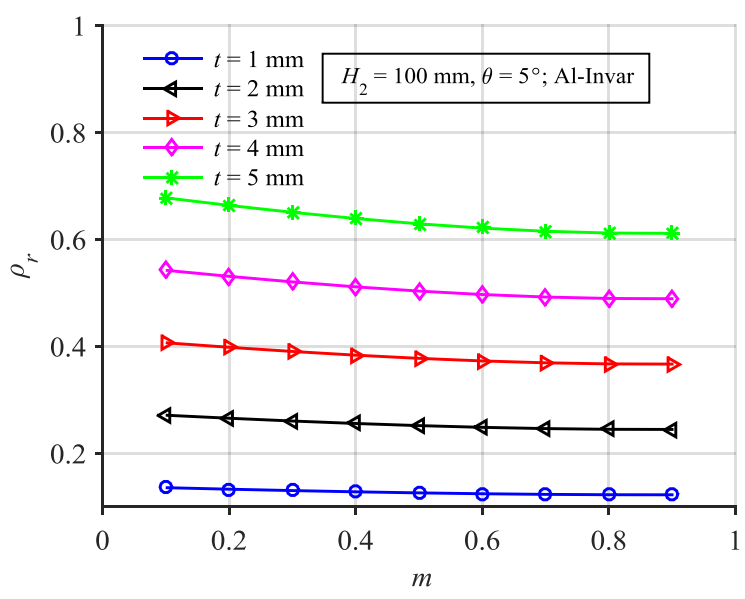

(d)

Fig. 9 Variations of the properties of metamaterial \# 2 with $H_{3}$ for five values of $t$ : (a) the effective Poisson's ratio $v$, (b) the normalized effective CTE $\alpha^{*}\left(\equiv \alpha / \alpha_{A l}\right)$, (c) the normalized effective Young's modulus $E^{*}\left(\equiv E / E_{A l}\right)$, and (d) the relative density $\rho_{r}$. Here $m \equiv H_{3} / M$. 
It is observed from Figs. 9(a) and 9(b) that $H_{3}$ has a large effect on $v$ and $\alpha^{*}$. From Fig. 9(a), it is seen that for each value of $t$ other than $t=1 \mathrm{~mm}, v$ decreases monotonically with the increase of $H_{3}$, and for a given value of $H_{3}, v$ decreases as $t$ increases. However, the effect of $H_{3}$ on $\alpha^{*}$ is different from that on $v$. Figure 9(b) shows that when the strut thickness $t=5 \mathrm{~mm}$, no negative $\alpha^{*}$ can be obtained, and the smallest $\alpha^{*}$ (around 0.01 ) is reached when $m=H_{3} / M=0.5$. However, when $t$ becomes smaller, the range and value of attainable negative $\alpha^{*}$ are getting larger: when $t=4 \mathrm{~mm}, \alpha^{*}$ is negative in the range of $0.4<m<0.6$ and reaches the maximum of -0.0085 ; when $t=3 \mathrm{~mm}, \alpha^{*}$ is negative in the range of $0.34<m<0.7$ and reaches the maximum of -0.0352 ; when $t=2 \mathrm{~mm}, \alpha^{*}$ is negative in the range of $0.3<m<0.76$ and reaches the maximum of -0.0805 ; when $t=1 \mathrm{~mm}, \alpha^{*}$ is negative in the range of $0.26<m<0.86$ and reaches the maximum of -0.1668 . Furthermore, Fig. $9(\mathrm{c})$ reveals that the effective Young's modulus $E^{*}$ increases as $H_{3}$ increases for each value of $t$. Also, increasing $t$ leads to the increase of $E^{*}$, as expected. In addition, Fig. 9(d) shows that the relative density $\rho_{r}$ decreases only slightly with the increase of $\mathrm{H}_{3}$, while it enlarges as $t$ increases.

Figure 10 displays how the effective PR, CTE, Young's modulus, and the relative density change with the parameter $H_{3}$ and the re-entrant angle $\theta$.

From Fig. 10(a), it can be observed that the lattice structure of metamaterial \# 2 cannot yield any negative PR value for the specified geometrical parameters and material combination. Instead, this lattice structure exhibits a large range of positive PR values from 0.1665 to around 1.0143. When $H_{3}$ increases, the PR decreases and reaches its minimum near $H_{3} / M=0.9$ in each case with a different value of $\theta$. From Fig. 10(b), it is seen that no negative CTE value can be obtained for the metamaterial with $\theta=35^{\circ}$ when $m\left(\equiv H_{3} / \mathrm{M}\right)$ varies from 0.1 to 0.9 . However, 
for the metamaterial with $\theta=25^{\circ}$, negative CTE values can be obtained when $0.65<m<0.9$. For the metamaterial with $\theta=15^{\circ}$, negative CTE values become possible when $0.4<m<0.85$ and the maximum negative CTE is reached when $m$ is around 0.7. However, after this point, the normalized CTE, $\alpha^{*}$, jumps from -0.1022 to 0.0486 at $m=0.9$. For the metamaterial with $\theta=5^{\circ}$, the transition point from positive to negative CTE is around $m=0.3$, and the maximum negative CTE is reached around $m=0.6$.

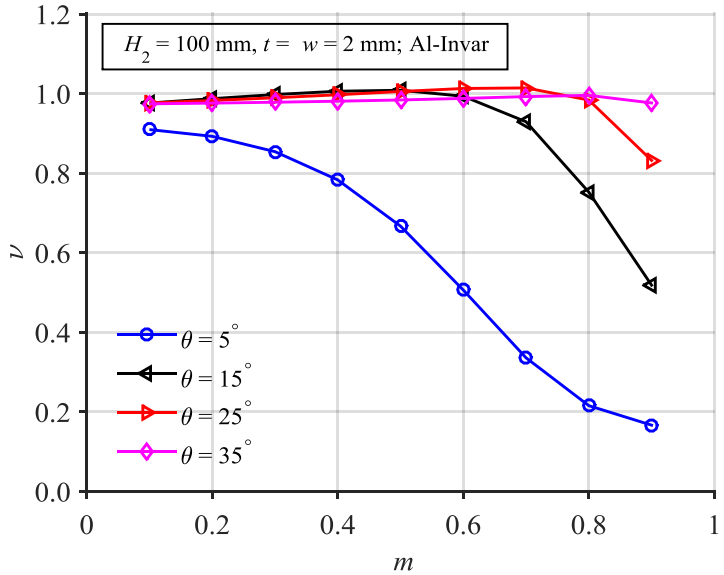

(a)

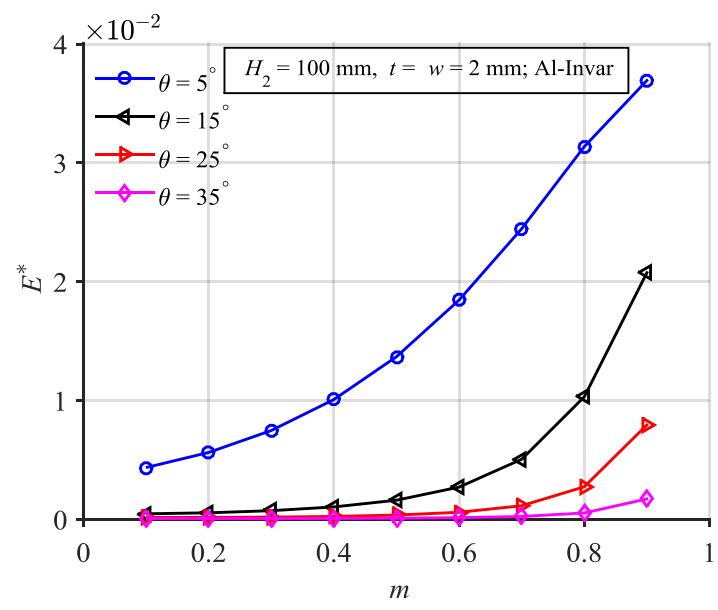

(c)

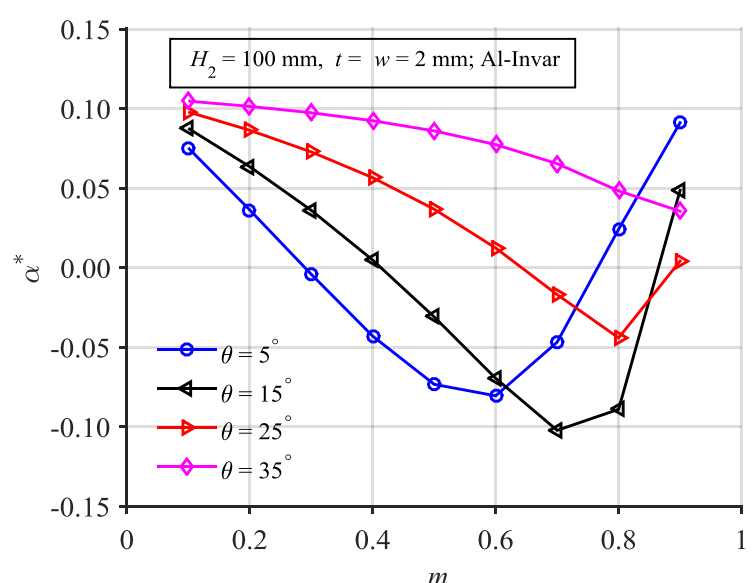

(b)

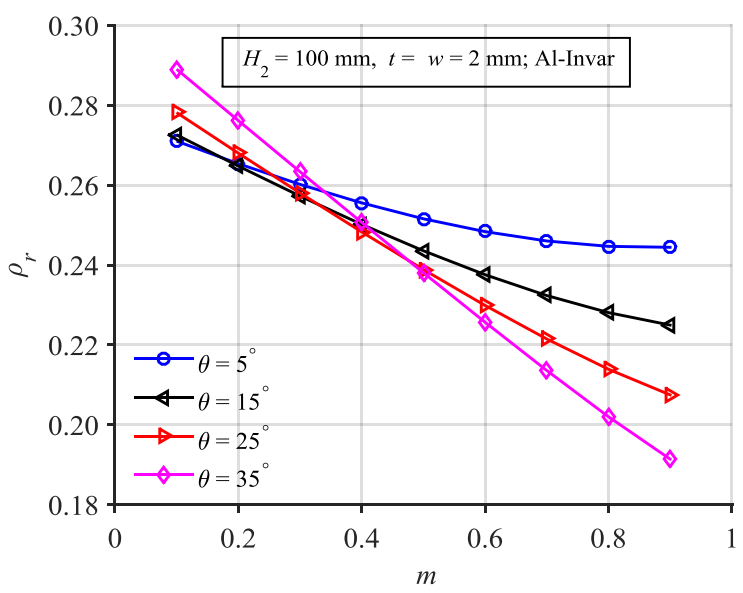

(d)

Fig. 10 Variations of the properties of metamaterial \# 2 with $\mathrm{H}_{3}$ for four different values of $\theta$ : (a) the effective Poisson's ratio $v$, (b) the normalized effective CTE $\alpha^{*}\left(\equiv \alpha / \alpha_{A l}\right)$, (c) the normalized effective Young's modulus $E^{*}\left(\equiv E / E_{A l}\right)$, and (d) the relative density $\rho_{r}$. Here $m \equiv H_{3} / M$.

From Fig. $10(\mathrm{c})$, it is observed that the effective Young's modulus $E^{*}$ increases as $H_{3}$ 
increases for each value of $\theta$ but it decreases considerably with the increase of $\theta$ for a given value of $H_{3}$. A comparison of the lattice structures with $\theta=5^{\circ}$ and $35^{\circ}$ shows that the difference is significantly large, with $E^{*}=3.6933 \times 10^{-2}$ for the former and $E^{*}=0.1722 \times 10^{-2}$ for the latter when $m=0$.9. Furthermore, Fig. 10(d) shows that the relative density $\rho_{r}$ decreases with the increase of $H_{3}$ but does not exhibit a monotonic trend as $\theta$ increases. In summary, when $\theta$ is small, the lattice structure of metamaterial \# 2 can attain a negative CTE, a large positive PR and a high Young's modulus. By choosing $\theta$ and $H_{3}$ properly, this metamaterial can be tailored to exhibit negative CTE values and large positive PR values.

Figure 11 shows the variations of $v, \alpha^{*}, E^{*}$ and $\rho_{r}$ with $\theta$ for metamaterial \# 2 with three different material combinations.

From Fig. 11(a), it can be observed that with the increase of $\theta, v$ remains almost unchanged after $\theta$ becomes larger than $20^{\circ}$. From Fig. 11(b), it is seen that only the lattice structure with the Al-Invar combination displays negative CTE values when $\theta$ is less than $23.5^{\circ}$, while the other two material combinations cannot result in any negative CTE value over the entire range of $\theta$ even though the metamaterial with the St-Invar combination exhibits near-zero CTE values when $\theta$ is less than $15^{\circ}$. A comparison of Figs. 11(a) and 11(b) shows that the effect of the material combination on $v$ is opposite to that on $\alpha^{*}$. That is, $v$ changes from the largest to the smallest, while $\alpha^{*}$ goes from the smallest to the largest, when the material combination varies in the order of Al-Invar, St-Invar and Al-St. The variation of the effective Young's modulus with $\theta$ is displayed in Fig. 11(c), which shows that $E^{*}$ is the highest for the metamaterial with the Al-St combination and the lowest for that with the At-Invar combination, with $E^{*}$ for the metamaterial with the St-Invar combination being in the middle. Furthermore, it is seen from Eq. 11(c) that $E^{*}$ is greatly reduced with the increase of $\theta$ for each of the three material combinations. Figure 11(d) 
displays how the relative density $\rho_{r}$ changes with $\theta$. Clearly, it is observed from Fig. 11(d) that $\rho_{r}$ decreases as $\theta$ increases for all of the three material combinations considered. Also, $\rho_{r}$ is the largest for the metamaterial with the Al-Invar combination and the smallest for that with the StInvar combination, which is the same order as that exhibited by metamaterial \# 1 (see Fig. 8(d)).

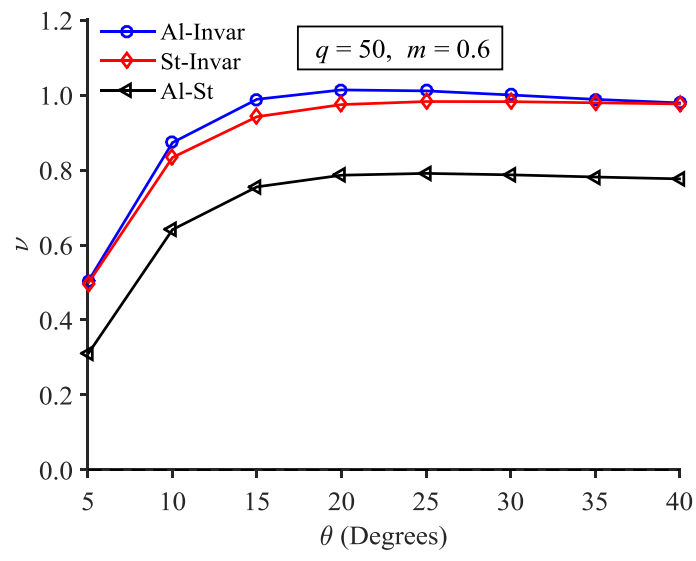

(a)

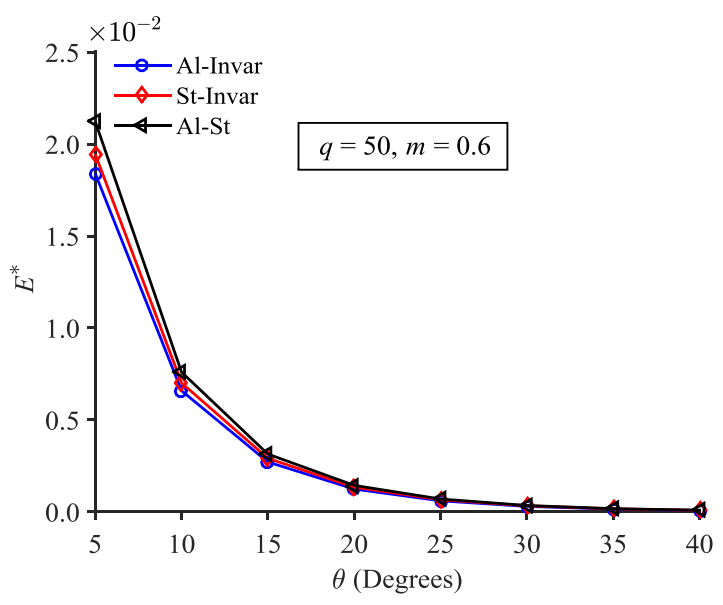

(c)

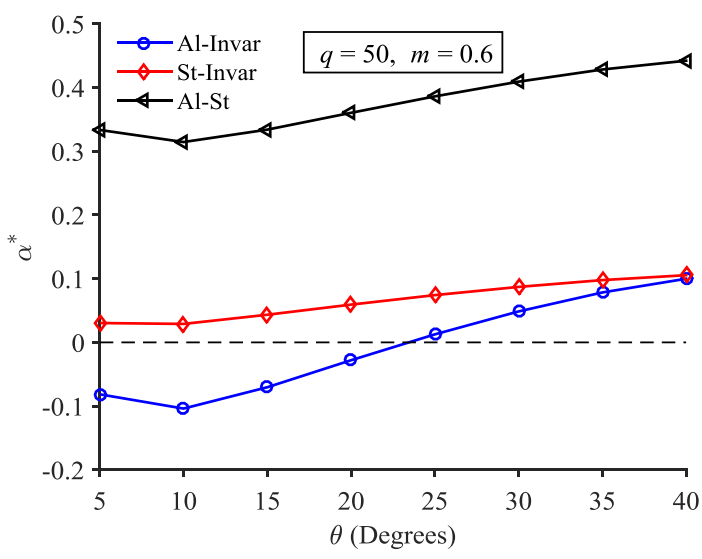

(b)

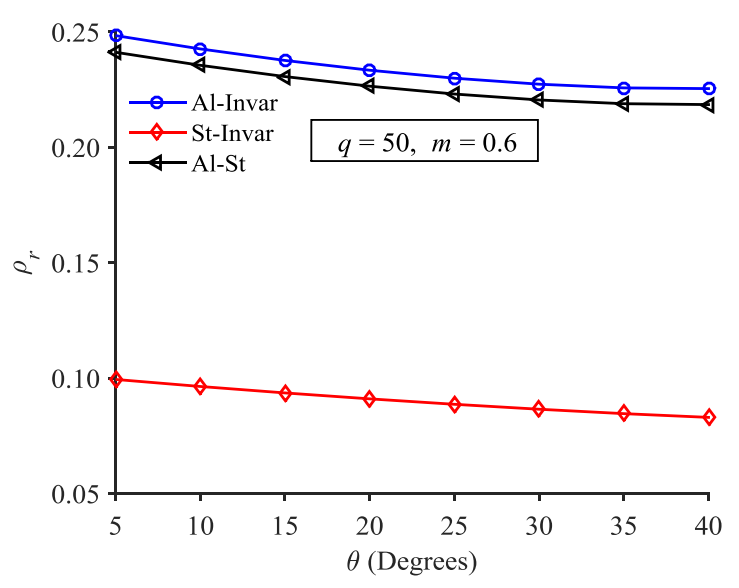

(d)

Fig. 11 Variations of the properties of metamaterial \# 2 with $\theta$ for three material combinations:

(a) the effective Poisson's ratio $v$, (b) the normalized effective CTE $\alpha^{*}\left(\equiv \alpha / \alpha_{A l}\right)$, (c) the normalized effective Young's modulus $E^{*}\left(\equiv E / E_{A l}\right)$, and (d) the relative density $\rho_{r}$. The crosssection of each strut is square with $t=w=2 \mathrm{~mm}$.

\subsection{Metamaterial \# 3}

It can be seen from Fig. 1 that metamaterial \# 3 is modified from the design for metamaterial \# 2 by adding four struts to the outskirt of its unit cell. As a result, $H_{1}$ is also involved in 
metamaterial \# 3 in addition to the other three parameters $H_{2}, H_{3}$ and $\theta$.

Figure 12 shows the variations of the effective PR, CTE, Young's modulus, and the relative density with the parameter $H_{3}$ and re-entrant angle $\theta$. The numerical values of $v, \alpha^{*}$ and $E^{*}$ are obtained from Eqs. (7)-(9) after each finite element simulation based on the unit cell shown in Fig. 2(c) is completed. The values for $\rho_{r}$ are computed using the formula for metamaterial \# 3 given in Table 2.

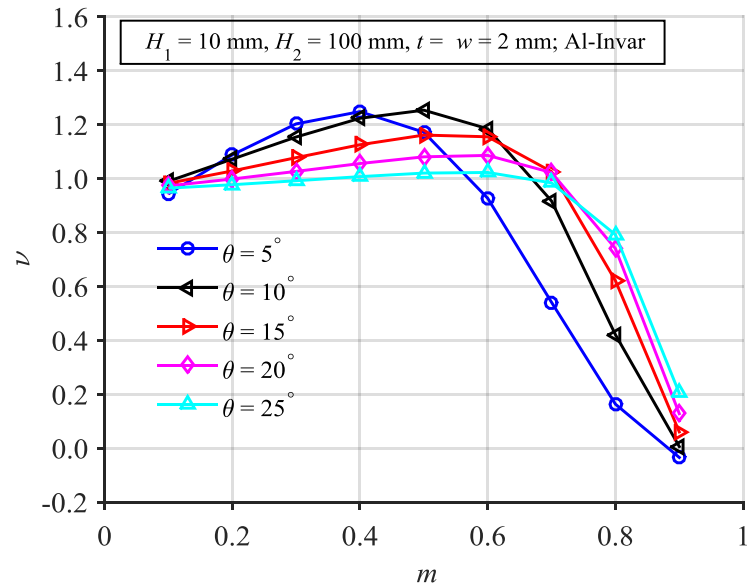

(a)

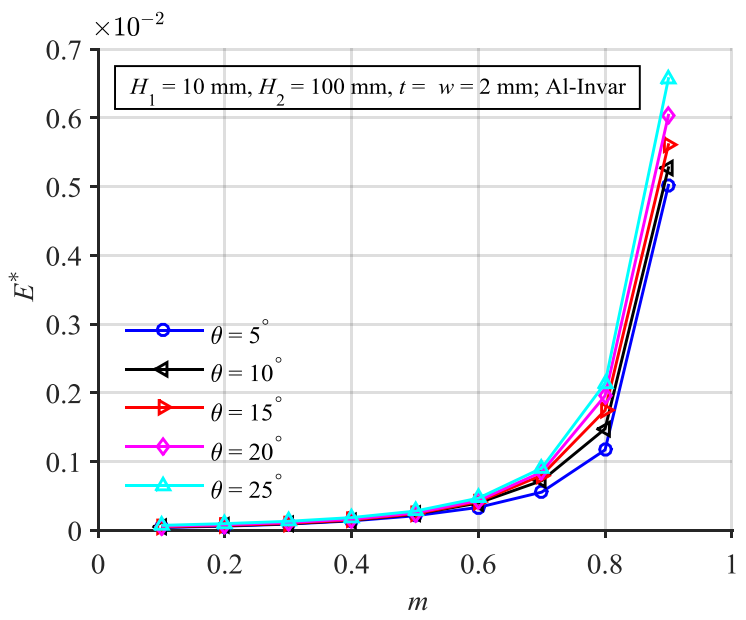

(c)

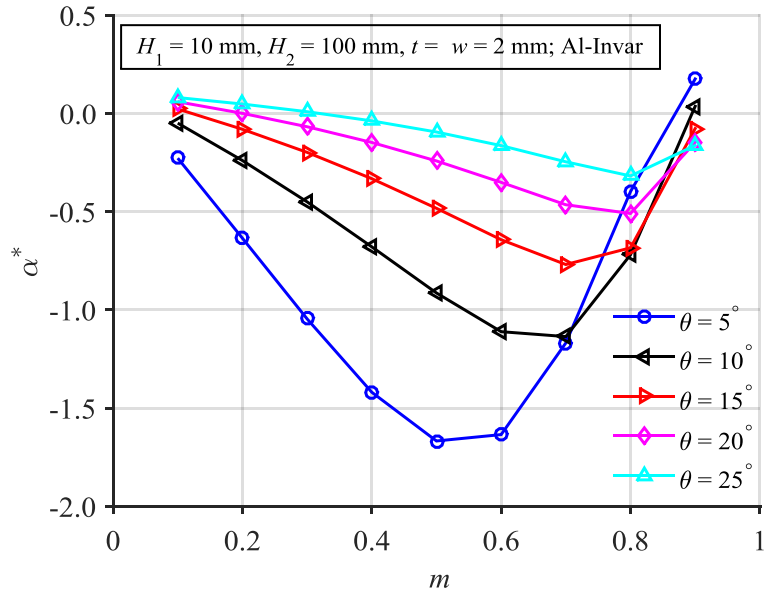

(b)

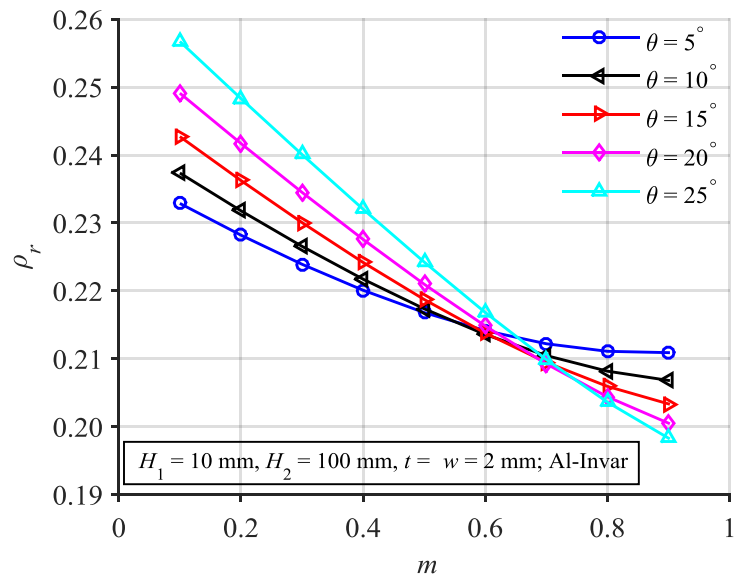

(d)

Fig. 12 Variations of the properties of metamaterial \# 3 with $H_{3}$ for five different values of $\theta$ : (a) the effective Poisson's ratio $v,(\mathrm{~b})$ the normalized effective CTE $\alpha^{*}\left(\equiv \alpha / \alpha_{A l}\right)$, (c) the normalized effective Young's modulus $E^{*}\left(\equiv E / E_{A l}\right)$, and (d) the relative density $\rho_{r}$. Here $m \equiv H_{3} / M$.

From Figs. 12(a) and 12(b), it is seen that the effects of changing the locations of the struts 
with the higher thermal expansion (through adjusting $H_{3}$ ) on the effective Poisson's ratio $v$ and CTE $\alpha^{*}$ are different. Figure 12(a) shows that the minimum value of $v$, which is close to zero, is obtained when $\mathrm{H}_{3}$ approaches its maximum limit. Also, $v$ increases to a maximum value and then decreases with the increase of $H_{3}$ for each value of $\theta$. It is observed from Fig. 12(b) that the maximum value of $\alpha^{*}$ (in magnitude) is reached when $m \equiv H_{3} / \mathrm{M}=0.5$ for $\theta=5^{\circ}, m=0.7$ for $\theta=$ $10^{\circ}, m=0.7$ for $\theta=15^{\circ}, m=0.8$ for $\theta=20^{\circ}$, and $m=0.8$ for $\theta=25^{\circ}$. Meanwhile, for each value of $\theta$, the trend of $\alpha^{*}$ changing with $H_{3}$ in the first and second half regions of $H_{3}$ is opposite to each other: in the first half $\alpha^{*}$ decreases with $H_{3}$, but in the second half $\alpha^{*}$ increases with $H_{3}$. Therefore, $H_{3}$ should be combined with $\theta$ to obtain desired $\alpha^{*}$ values. It is seen from Fig. 12(c) that the effective Young's modulus $E^{*}$ increases considerably as $H_{3}$ approaches its maximum value. Figure 12(d) shows that the relative density $\rho_{r}$ decreases with $H_{3}$ for each value of $\theta$. Therefore, using the maximum possible value for $H_{3}$ will lead to a large Young's modulus and a near-zero Poisson's ratio, but negative CTE cannot be attained simultaneously for the Al-Invar material combination considered.

The effects of $\mathrm{H}_{2}$ and $t$ on the properties of metamaterial \# 3 are displayed in Figs. 13(a)13(d). It is clearly observed from Figs. 13(a) and 13(b) that these two parameters have opposite effects on the effective Poisson's ratio and CTE. Increasing $H_{2}$ results in a large positive Poisson's ratio value and a large negative CTE value (in magnitude). Meanwhile, increasing the strut thickness $t$ renders the Poisson's ratio to approach zero and makes the CTE value less negative or even positive. Figure 13(c) shows that the effective Young's modulus $E^{*}$ decreases with the increase of $H_{2}$ and enlarges as $t$ increases. In addition, the relative density $\rho_{r}$ decreases with $\mathrm{H}_{2}$ for each value of $t$ and becomes larger as $t$ gets larger. Therefore, to obtain a lattice structure of metamaterial \# 3 with a small or near-zero PR and CTE, thicker struts should be used 
together with a smaller $H_{2}$ (than $H_{1}$ ), which will also ensure a sufficiently high Young's modulus (see Fig. 13(c)).

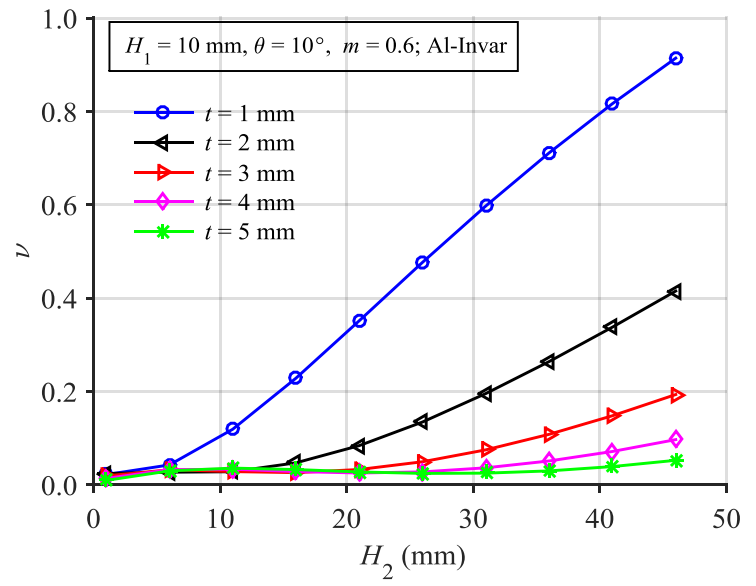

(a)

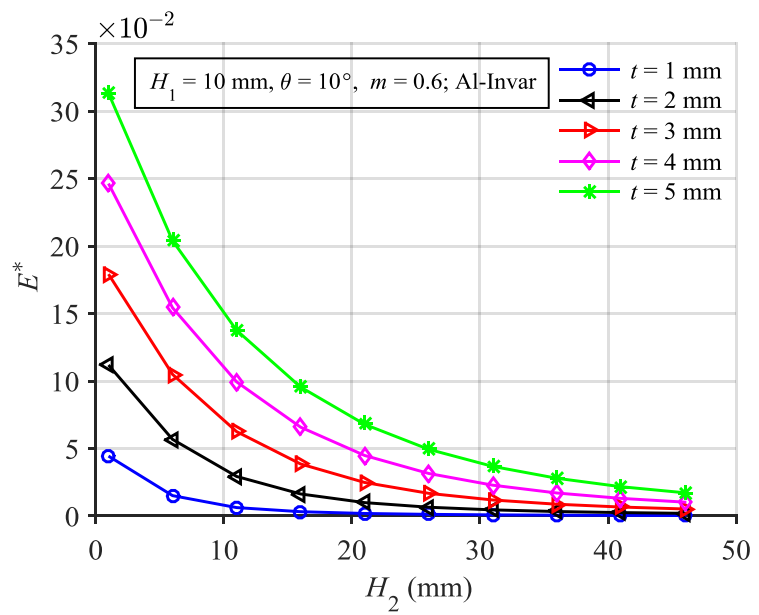

(c)

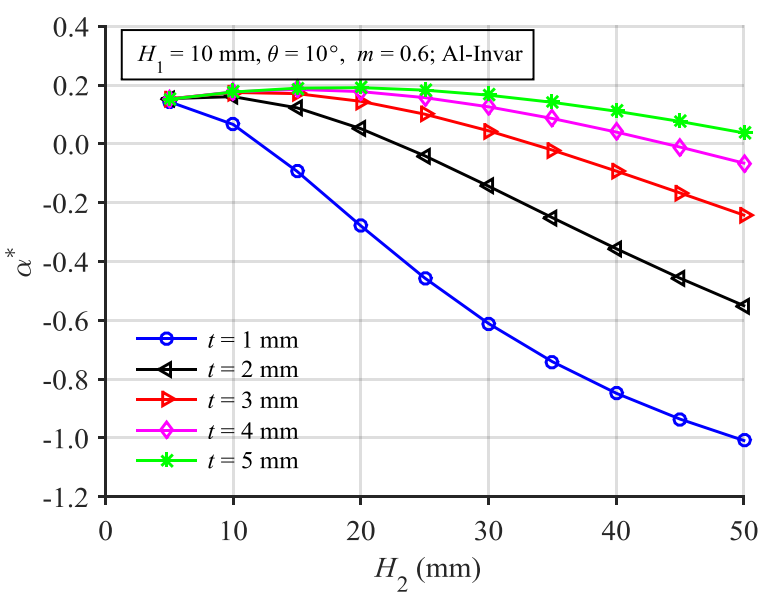

(b)

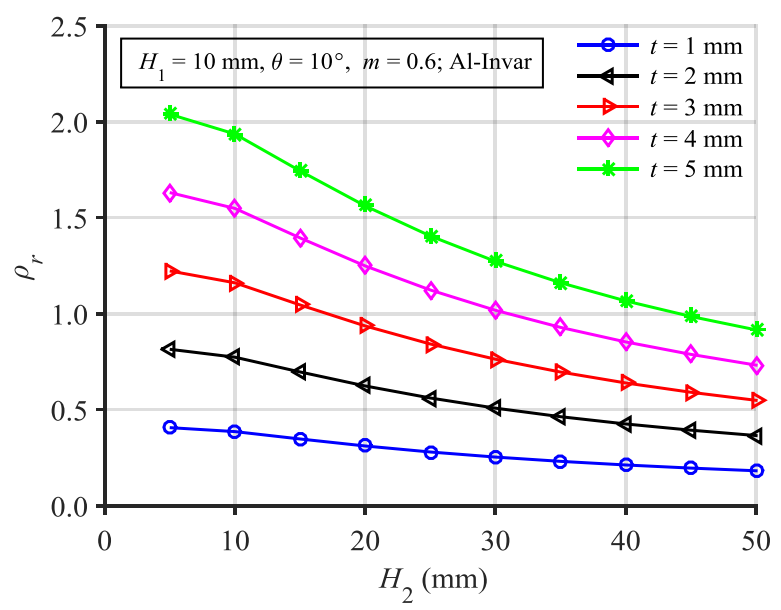

(d)

Fig. 13 Variations of the properties of metamaterial \# 3 with $\mathrm{H}_{2}$ for five different values of $t$ : (a) the effective Poisson's ratio $v$, (b) the normalized effective CTE $\alpha^{*}$ ( $\equiv \alpha / \alpha_{A l}$ ), (c) the normalized effective Young's modulus $E^{*}\left(\equiv E / E_{A l}\right)$, and (d) the relative density $\rho_{r}$. Here $m \equiv H_{3} / M$.

Figure 14 displays the variations of $v, \alpha^{*}, E^{*}$ and $\rho_{r}$ with $\theta$ for metamaterial \# 3 with three different material combinations.

It is observed from Figs. 14(a) and 14(b) that changing the material combination from AlInvar to St-Invar to Al-St leads to the variations of the values of $v$ and $\alpha^{*}$ and $v$ in opposite orders, which is the same as that observed in Sections 4.1 and 4.2 for metamaterials \# 1 and \# 2, 
respectively. It is seen from Fig. 14(a) that only when $\theta$ is smaller than $1^{\circ}$, negative values may be attained for the effective Poisson's ratio $v$. With the increase of $\theta, v$ first increases until reaching its maximum value and then decreases. Figure 14(b) shows that the lattice structure with the Al-Invar combination can attain negative CTE values when $\theta$ is in the range of $1^{\circ}$ to $33^{\circ}$, while the lattice structures with the St-Invar and Al-St combinations can do so only when $2^{\circ}<\theta$ $<25^{\circ}$ and $2.5^{\circ}<\theta<15^{\circ}$, respectively. It is observed from Fig. 14(c) that the effective Young's modulus $E^{*}$ is the highest for the Al-St combination and the lowest for the Al-Invar combination. Also, $E^{*}$ increases monotonically with $\theta$. Figure $14(\mathrm{~d})$ reveals that the relative density $\rho_{r}$ does not display a monotonic variation as $\theta$ increases. This is different from that observed in Sections 4.1 and 4.2 for metamaterials \# 1 and \# 2 (see Fig. 8(d) and 11(d)).

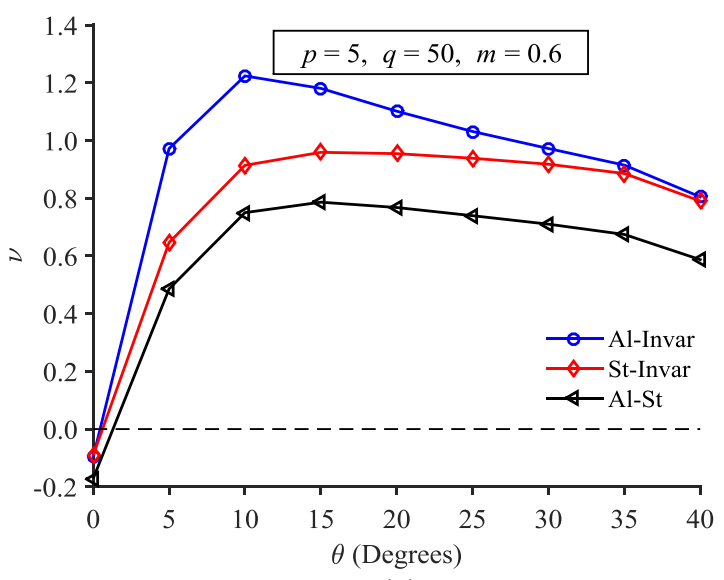

(a)

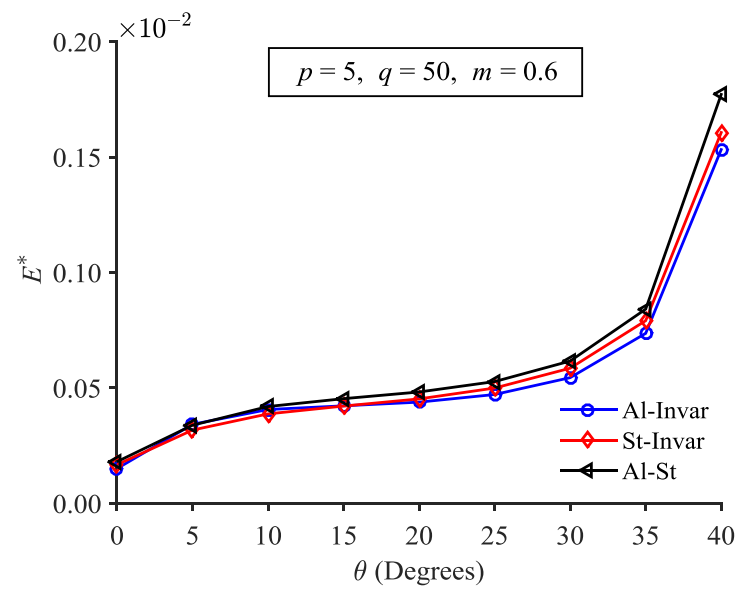

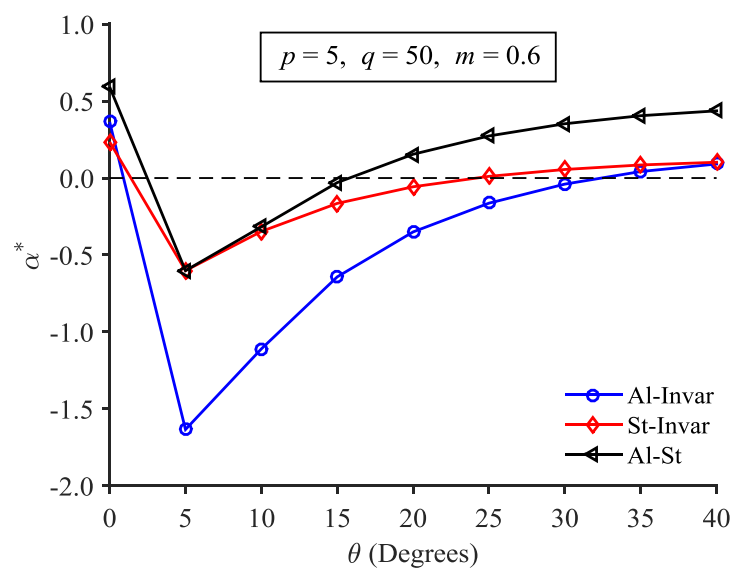

(b)

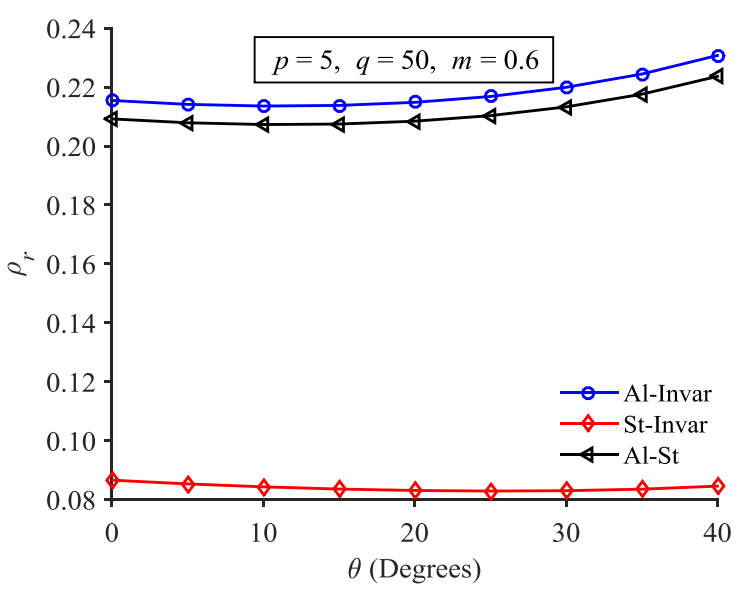


(c)

(d)

Fig. 14 Variations of the properties of metamaterial \# 3 with $\theta$ for three material combinations:

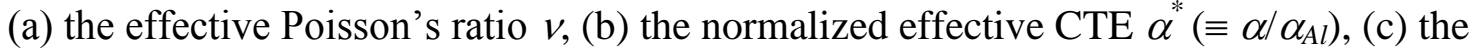
normalized effective Young's modulus $E^{*}\left(\equiv E / E_{A l}\right.$ ), and (d) the relative density $\rho_{r}$. The cross-

4.4 Metamaterial \# 4 section of each strut is square with $t=w=2 \mathrm{~mm}$.

As shown in Fig. 1, metamaterial \# 4 can be obtained from combining the lattice structures for metamaterials \# 1 and \# 2. As a result, all four geometrical parameters are involved in this design and their effects on the properties of metamaterial \# 4 need to be studied.

Figure 15 displays how the effective PR, CTE, Young's modulus, and the relative density change with $\mathrm{H}_{3}$ and $\theta$. The numerical values of $v, \alpha^{*}$ and $E^{*}$ are obtained from Eqs. (7)-(9) after each finite element simulation based on the unit cell shown in Fig. 2(d) is completed. The values for $\rho_{r}$ are computed using the formula for metamaterial \# 4 given in Table 2.

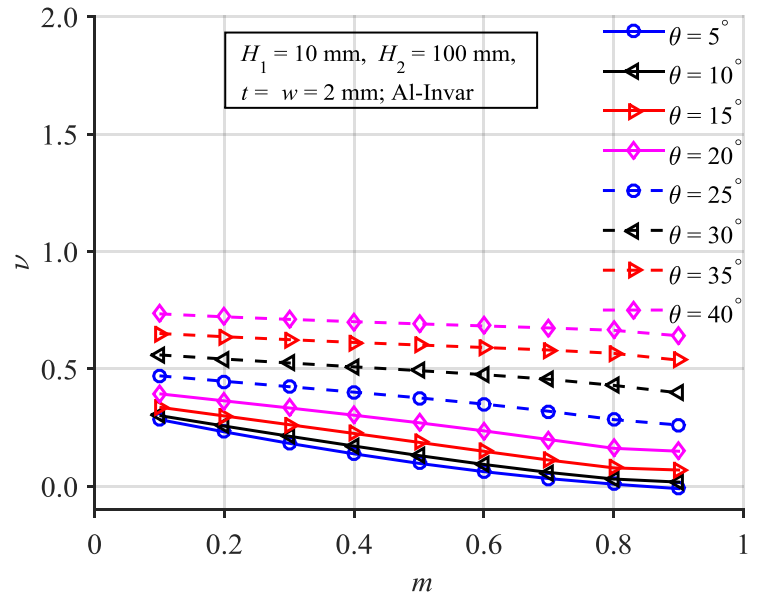

(a)

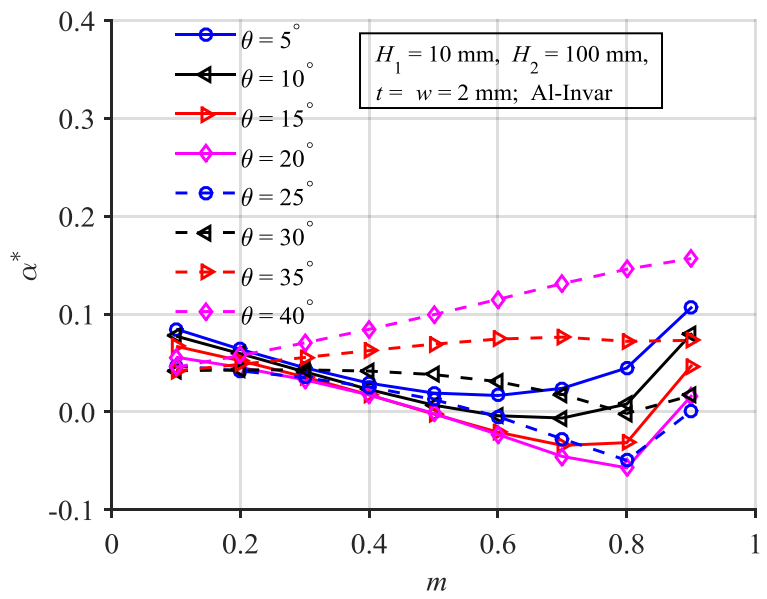

(b) 


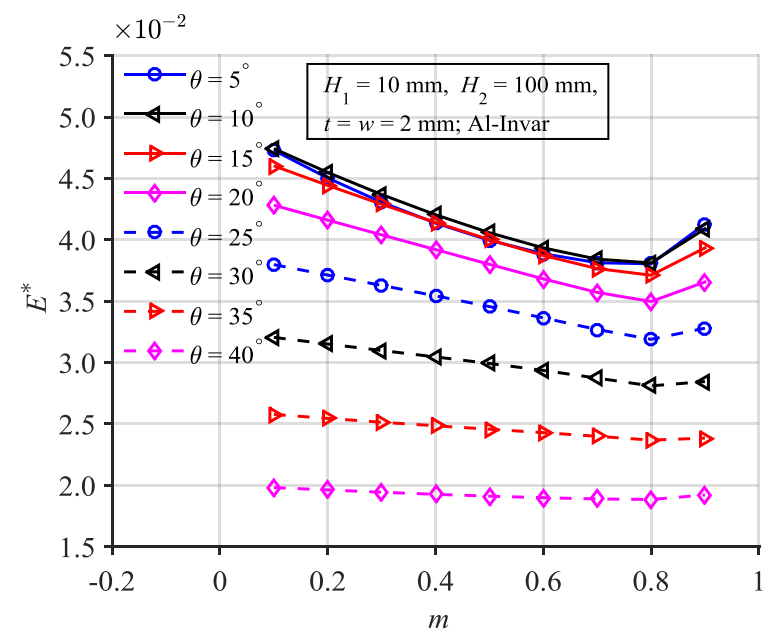

(c)

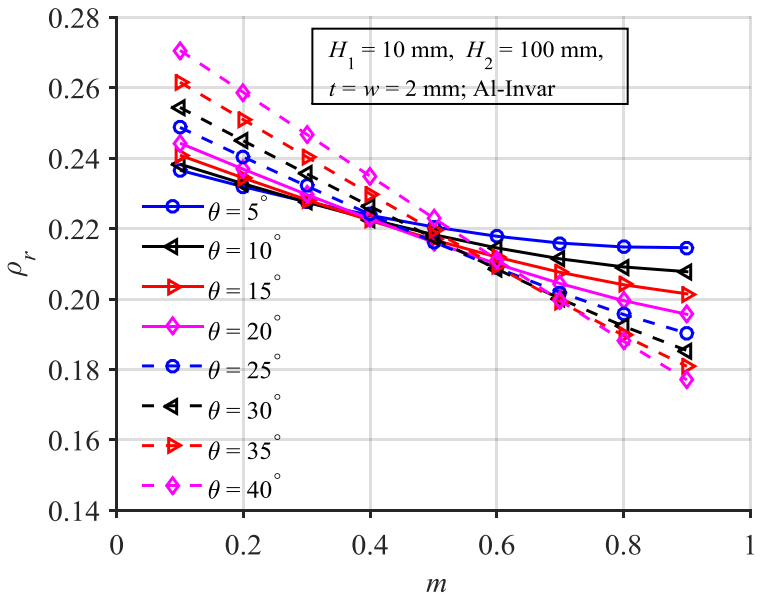

(d)

Fig. 15 Variations of the properties of metamaterial \# 4 with $H_{3}$ for eight different values of $\theta$ : (a) the effective Poisson's ratio $v,(\mathrm{~b})$ the normalized effective CTE $\alpha^{*}\left(\equiv \alpha / \alpha_{A l}\right)$, (c) the normalized effective Young's modulus $E^{*}\left(\equiv E / E_{A l}\right)$, and (d) the relative density $\rho_{r}$. Here $m \equiv H_{3} / M$.

It is seen from Fig. 15(a) that a smaller angle $\theta$ leads to a smaller $v$. Figure 15(b) shows that the effect of $\theta$ on $\alpha^{*}$ of metamaterial \# 4 does not display a clear and consistent trend. For the lattice structures of metamaterial \# 4 with $\theta=5^{\circ}, 35^{\circ}$ and $40^{\circ}$, no negative CTE can be attained over the entire range of $H_{3}$. However, for $\theta=10^{\circ}, 15^{\circ}, 20^{\circ}, 25^{\circ}$ and $30^{\circ}$, the metamaterial with a negative CTE can be obtained when $H_{3}$ exceeds a specific transition point. Therefore, in order to obtain a negative CTE for metamaterial \# 4, the value of $\theta$ should not be $5^{\circ}, 35^{\circ}$ or $40^{\circ}$. It appears that $\theta=10^{\circ}$ is a good design option if both near-zero PR and CTE are desired. Figure 15(c) shows how the effective Young's modulus $E^{*}$ changes with the geometrical parameters $H_{3}$ and $\theta$. It can be seen that $\theta$ has a much more pronounced effect on $E^{*}$ than $H_{3}$. For the relative density $\rho_{r}$, it is observed from Fig. 15(d) that $\rho_{r}$ decreases with the increase of $H_{3}$ for each value of $\theta$.

The effects of $H_{2}$ and $t$ on the properties of metamaterial \# 4 are shown in Figs. 16(a)-16(d). For the effective Poisson's ratio $v$, Fig. 16(a) shows that with the increase of $H_{2}, v$ does not increase monotonically. Moreover, increasing $t$ leads to larger $v$. From Fig. 16(b), it is seen that increasing $H_{2}$ results in smaller $\alpha^{*}$. Also, reducing the strut thickness $t$ leads to a decrease in $\alpha^{*}$, which is the same 
variation trend as that exhibited by $v$. In addition, it is seen from Fig. 16(c) that increasing $H_{2}$ reduces $E^{*}$. Furthermore, Fig. 16(c) shows that $E^{*}$ increases with the increase of $t$. It is observed from Fig. 16(d) that $\rho_{r}$ decreases with the increase of $H_{2}$ but enlarges as $t$ increases.

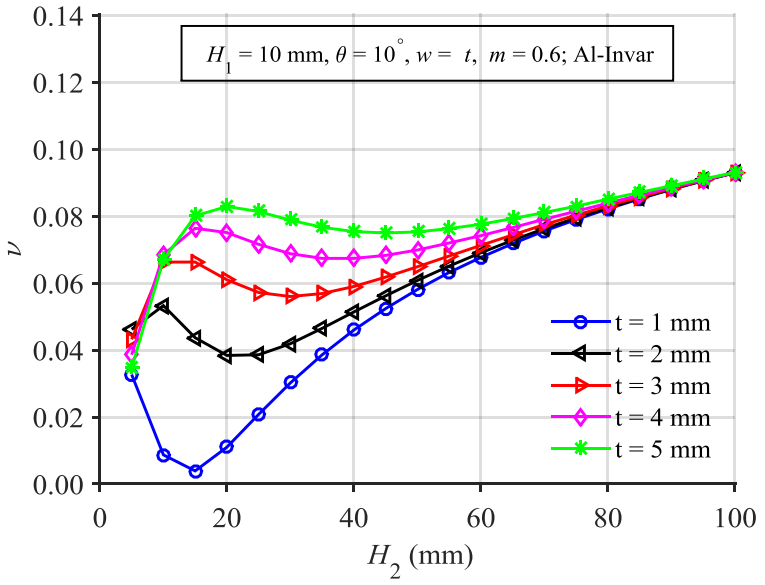

(a)

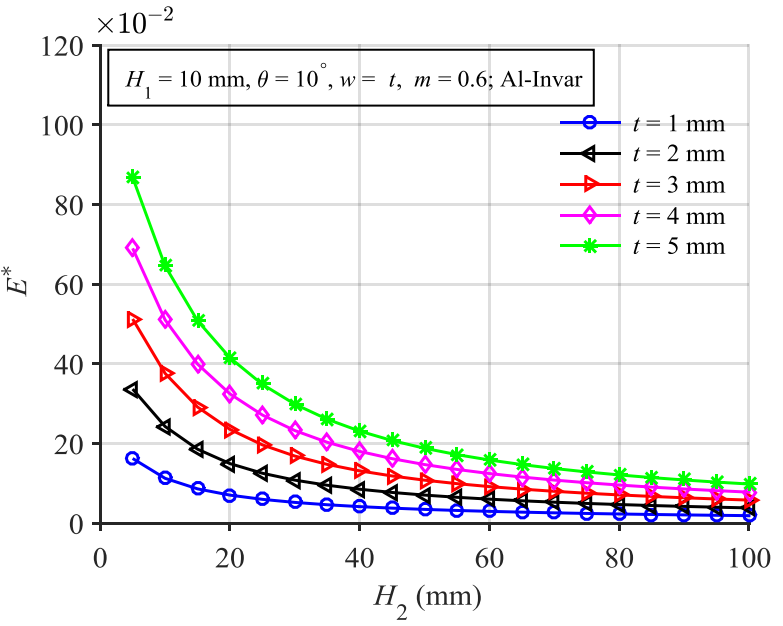

(c)

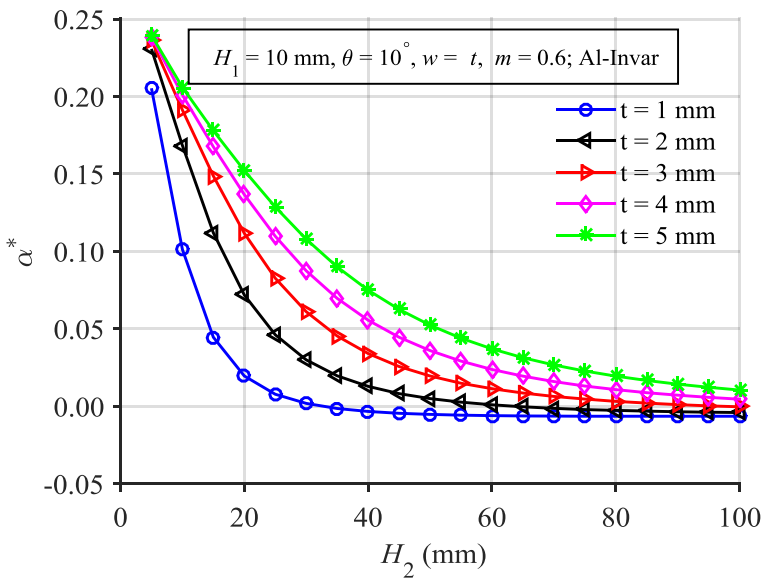

(b)

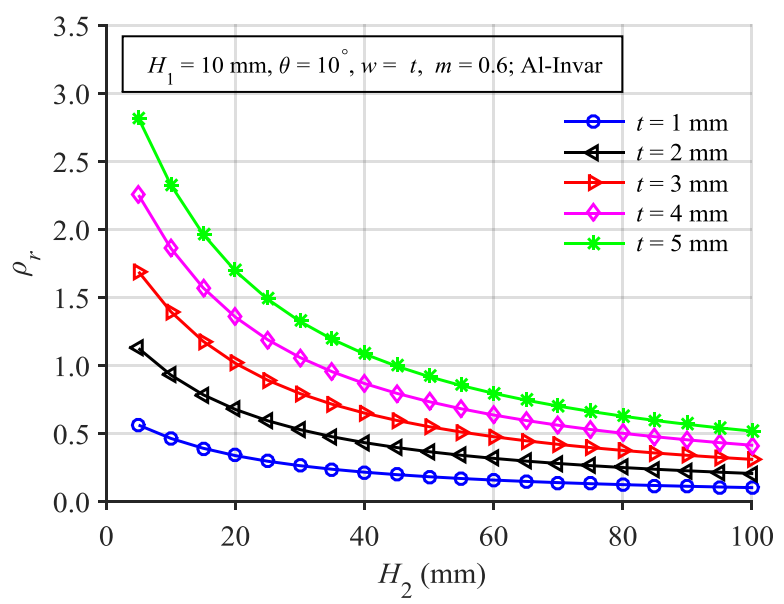

(d)

Fig. 16 Variations of the properties of metamaterial \# 4 with $\mathrm{H}_{2}$ for five different values of $t$ : (a) the effective Poisson's ratio $v$, (b) the normalized effective CTE $\alpha^{*}$ ( $\equiv \alpha / \alpha_{A l}$ ), (c) the normalized effective Young's modulus $E^{*}\left(\equiv E / E_{A l}\right)$, and (d) the relative density $\rho_{r}$

Figures 17(a) and 17(b) display the variations of the effective PR and CTE with $\theta$ for three different material combinations.

From Fig. (17a), it is observed that a negative or near-zero PR can be obtained when $\theta$ is not large (less than $15^{\circ}$ for all the three material combinations). It is seen from Fig. 17(b) that 
metamaterial \# 4 with the Al-St and St-Invar combinations cannot attain a negative or near-zero CTE, while that with the Al-Invar combination can exhibit a negative CTE when $10^{\circ}<\theta<25^{\circ}$. Figure 17(c) shows that $E^{*}$ decreases with the increase of $\theta$ for each of the three material combinations, while Fig. 17(d) reveals that $\rho_{r}$ does not change monotonically as $\theta$ increases.

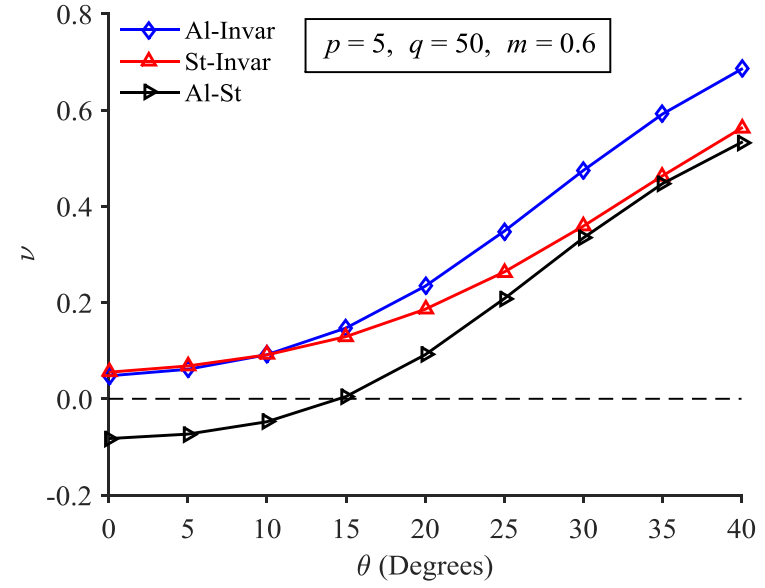

(a)

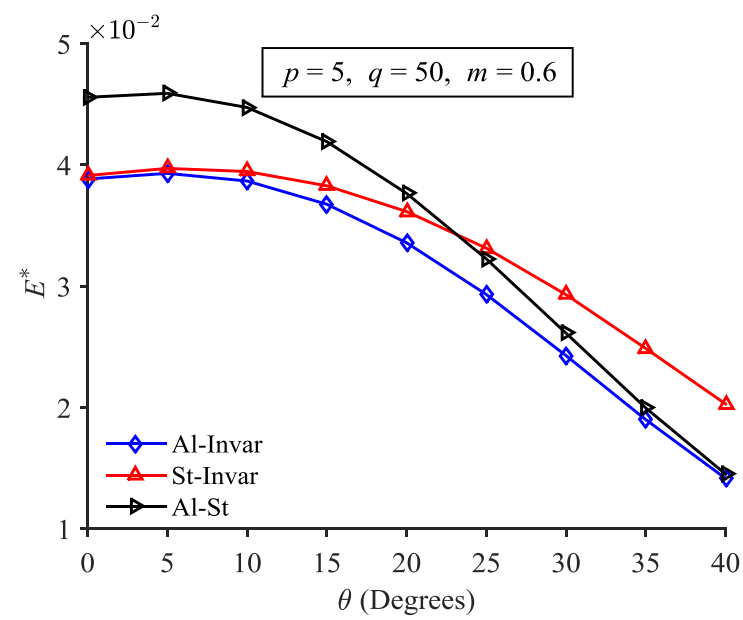

(c)

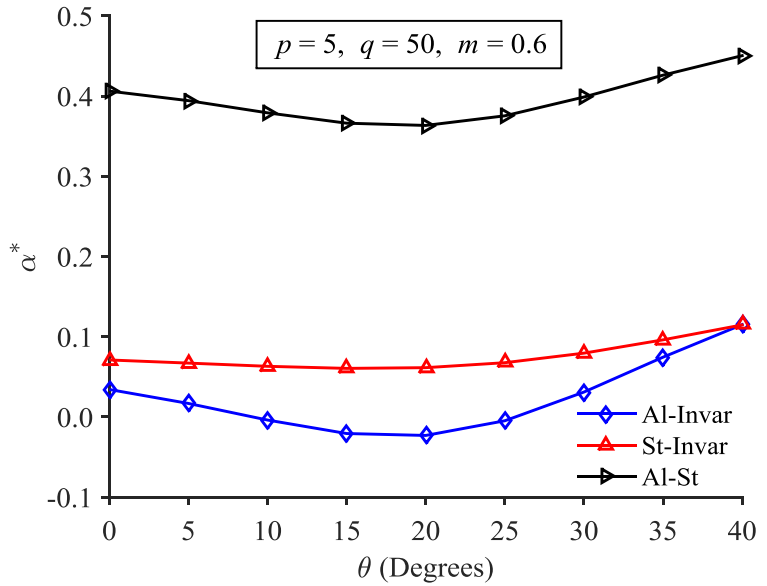

(b)

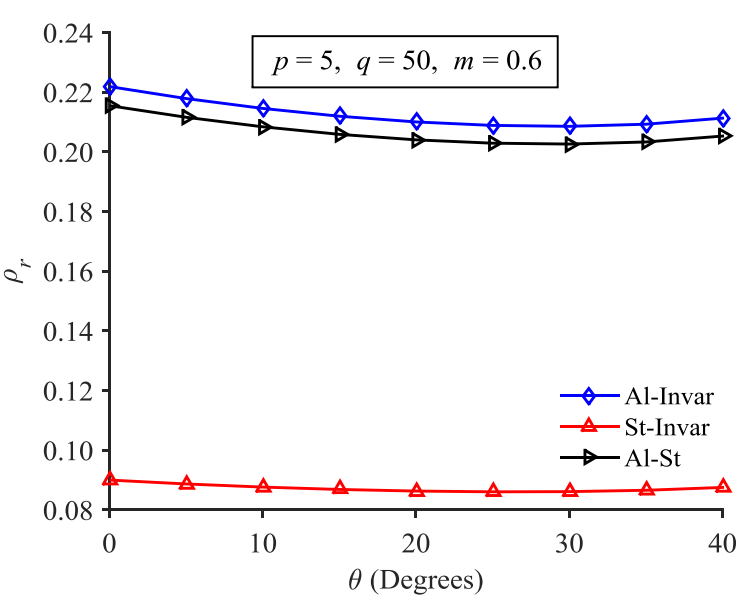

(d)

Fig. 17 Variations of the properties of metamaterial \# 4 with $\theta$ for three material combinations:

(a) the effective Poisson's ratio $v$, (b) the normalized effective CTE $\alpha^{*}$ ( $\left.\equiv \alpha / \alpha_{A l}\right)$, (c) the normalized effective Young's modulus $E^{*}\left(\equiv E / E_{A l}\right)$, and (d) the relative density $\rho_{r}$. The crosssection of each strut is square with $t=w=2 \mathrm{~mm}$.

From the parametric studies of the four designed metamaterials presented above, it is found that all the lattice structures considered can exhibit a negative CTE, the extent of which varies with the geometrical parameters and material combinations. However, not all of them can 
display a negative PR and/or a large Young's modulus. Without taking the Young's modulus into account, the four designed metamaterials with possible PR and CTE combinations are summarized in Table 3.

Table 3 Summary of possible PR and CTE combinations for the four metamaterials designed

\begin{tabular}{|c|c|c|c|}
\hline Metamaterial \# 1 & Metamaterial \# 2 & Metamaterial \# 3 & Metamaterial \# 4 \\
\hline & Positive PR and & Near-zero PR and & Positive PR and \\
Near-zero PR and & near-zero CTE; & near-zero CTE; \\
near-zero CTE; & Positive PR PR and & Positive PR and & Positive PR and \\
Negative PR and & negative CTE; & negative CTE; \\
negative CTE; & near-zero CTE; & Near-zero PR and & Near-zero PR and \\
Negative PR and & Positive PR and & positive CTE; & positive CTE; \\
positive CTE. & positive CTE. & Positive PR and & Positive PR and \\
& & positive CTE. & positive CTE. \\
\hline
\end{tabular}

\section{Comparison and Recommendation}

In order to further delineate the differences in the properties of the four metamaterials designed and to provide a quick comparison among the four designs., a bar chart is provided in Fig. 18 with the geometrical parameters $H_{1}=10 \mathrm{~mm}, H_{2}=100 \mathrm{~mm}, t=2 \mathrm{~mm}, w=2 \mathrm{~mm}$, and $\theta=20^{\circ}$. 


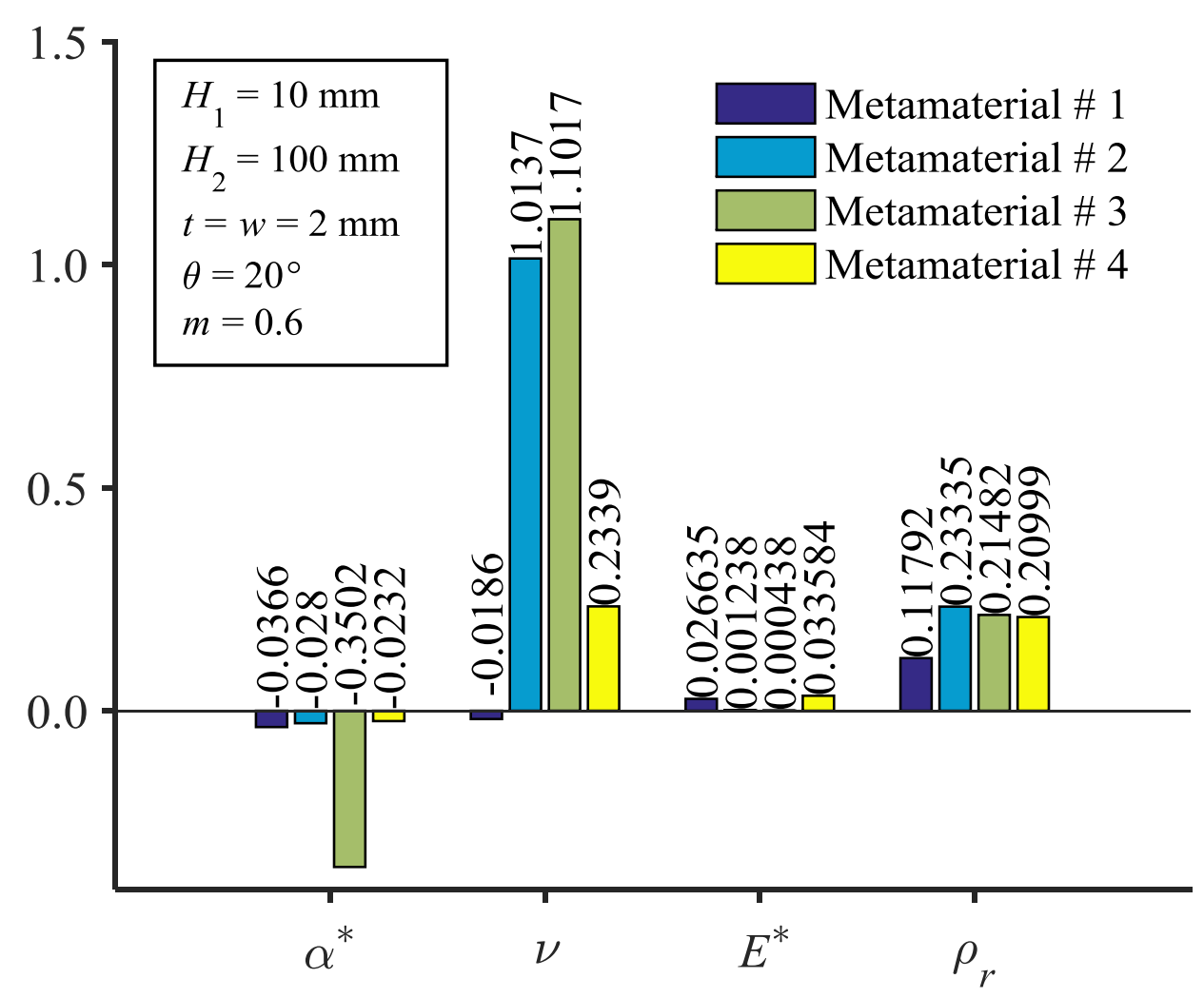

Fig. 18 Comparison among the four metamaterials designed. The vertical axis shows numerical values for each of the four non-dimensional properties listed along the horizontal axis.

It can be seen from Fig. 18 that under the given conditions, all of the four metallic metamaterials can exhibit a negative CTE, but only the first metamaterial can achieve a negative PR simultaneously. A positive PR can be attained for the other three metamaterials. For the effective Young's modulus, it is observed that metamaterial \# 3 possesses the smallest value, while metamaterial \# 4 has the largest one. The relative density of metamaterial \# 1 is smaller than that of metamaterial \# 2 or \# 3, but the stiffness of metamaterial \# 1 is larger than that of metamaterial \# 2 or \# 3. Therefore, in order to obtain the negative PR and CTE simultaneously with a good load-carrying capacity and a small relative density (so as to be lightweight), the metallic lattice structure of metamaterial \# 1 is recommended among the four designs. 


\section{Conclusion}

Four metallic metamaterials with tailorable mechanical properties are designed using bimaterial star-shaped re-entrant planar lattice structures. Three length parameters, one angle parameter and three material combinations are employed as design variables. The effects of the geometrical parameters and material combinations on the effective Poisson's ratio, coefficient of thermal expansion, Poisson's ratio, Young's modulus, and the relative density of each metamaterial are studied in detail using unit cell-based finite element simulations that employ periodic boundary conditions.

It is found that the metamaterials designed exhibit a unique combination of the mechanical properties that is not attainable from conventional materials. By appropriately selecting the geometrical parameters and material combination, metamaterials with desired properties can be designed. In particular, it is shown that metallic metamaterials with both a negative Poisson's ratio and a non-positive CTE can be obtained from the lattice structure of metamaterial \# 1.

\section{Acknowledgment}

The work reported here is funded by a grant from the U.S. National Science Foundation (NSF), Mechanics of Materials and Structures Program, with Dr. Kara Peters as the Director. This support is greatly appreciated. The authors also thank Prof. Antonio Ferreira and two anonymous reviewers for their encouragement and helpful comments on an earlier version of the paper.

\section{References}

Agnese, F. and Scarpa, F., 2014, Macro-composites with star-shaped inclusions for vibration damping in wind turbine blades, Composite Structures 108: 978-986. 
ANSYS, Inc., 2015, ANSYS Mechanical APDL Structural Analysis Guide, ANSYS, Inc., Canonsburg, PA, USA.

Ashby, M. F., 2006, The properties of foams and lattices, Philosophical Transactions of the Royal Society A 364: 15-30.

Berger, J., Mercer, C., McMeeking, R. M., and Evans, A. G., 2011, The design of bonded bimaterial lattices that combine low thermal expansion with high stiffness, Journal of the American Ceramic Society 94(s1): s42-s54

Chen, J., Hu, L., Deng, J. X. and Xing, X. R., 2015, Negative thermal expansion in functional materials: controllable thermal expansion by chemical modifications, Chemical Society Reviews 44: 3522-3567.

Chen, Y. Y., Jia, Z. A. and Wang, L. F., 2016, Hierarchical Honeycomb Lattice Metamaterials with Improved Thermal Resistance and Mechanical Properties, Composite Structures 152: $395-402$.

Corredor, A., Park, W. H., Cho, M. and Kim, Y.-S., 2013, Optomechanical analysis and testing of a fast steering secondary mirror prototype for the Giant Magellan Telescope, in Proceedings of the SPIE, Vol. 8836, pp. 88360U-1-88360U-14, International Society for Optics and Photonics.

Ding, Y. and Kovacevic, R., 2016, Feasibility study on 3-D printing of metallic structural material using robotized laser-based metal additive manufacturing, Journal of the Minerals, Metals and Materials Society (JOM) 68 (7): 1774-1779.

Gao, X.-L., 2015, A new Timoshenko beam model incorporating microstructure and surface energy effects, Acta Mechanica 226: 457-474.

Gatt, R., Attard, D., Farrugia, P. S., Azzopardi, K. M., Mizzi, L., Brincat, J. P. and Grima, J. N., 
2013, A realistic generic model for anti-tetrachiral systems, Physica Status Solidi (b) 250 (10): 2012-2019.

Gibson, L. J. and Ashby, M. F., 1997, Cellular Solids: Structure and Properties, 2nd edition, Cambridge University Press, Cambridge.

Gong, X. B., Huang, J., Scarpa, F., Liu, Y. J. and Leng, J. S., 2015, Zero Poisson's ratio cellular structure for two-dimensional morphing applications, Composite Structures 134: 384-392.

Greaves, G. N., Greer, A. L., Lakes, R. S. and Rouxel, T., 2011, Poisson's ratio and modern materials, Nature Materials 10(11): 823-837.

Grima, J. N., Farrugia, P. S., Gatt, R. and Zammit, V., 2007, A system with adjustable positive or negative thermal expansion, Proceedings of the Royal Society A 463 (2082): 1585-1596.

Grima, J. N., Jackson, R., Alderson, A. and Evans, K. E., 2000, Do zeolites have negative Poisson's ratios? Advanced Materials 12(24): 1912-1918.

Ha, C. S., Hestekin, E., Li, J. H., Plesha, M. E. and Lakes, R. S., 2015, Controllable thermal expansion of large magnitude in chiral negative Poisson's ratio lattices, Physica Status Solidi (b) 252(7): 1431-1434.

Harders, H., Hupfer, K. and Rösler, J., 2005, Influence of cell wall shape and density on the mechanical behaviour of 2D foam structures, Acta Materialia 53(5): 1335-1345.

Hopkins, J. B., Song, Y., Lee, H., Fang, N. X. and Spadaccini, C. M., 2016, Polytope sectorbased synthesis and analysis of microstructural architectures with tunable thermal conductivity and expansion, ASME Journal of Mechanical Design 138(5): 051401-1051401-10.

Huang, C. and Chen, L., 2016, Negative Poisson's ratio in modern functional materials, Advanced Materials 2016, DOI: 10.1002/adma.201601363. 
Lakes, R., 1987, Foam structures with a negative Poisson's ratio, Science 235(4792): 1038-1040.

Lakes, R., 1996, Cellular solid structures with unbounded thermal expansion, Journal of Material Science Letters 15(6): 475-477

Lakes, R., 2007, Cellular solids with tunable positive or negative thermal expansion of unbounded magnitude, Applied Physics Letters 90(22): 221905-1-221905-3.

Li, K., Gao, X.-L. and Roy, A. K., 2003, Micromechanics model for three-dimensional open-cell foams using a tetrakaidecahedral unit cell and Castigliano's second theorem, Composites Science and Technology 63(12): 1769-1781.

Li, K., Gao, X.-L. and Roy, A. K., 2005a, Micromechanical modeling of three-dimensional open-cell foams using the matrix method for spatial frames, Composites Part B: Engineering 36(3): 249-262.

Li, K., Gao, X.-L. and Subhash, G., 2005, Effects of cell shape and cell wall thickness variations on the elastic properties of two-dimensional cellular solids, International Journal of Solids and Structures 42(5-6): 1777-1795.

Li, K., Gao, X.-L. and Subhash, G., 2006, Effects of cell shape and strut cross-sectional area variations on the elastic properties of three-dimensional open-cell foams, Journal of the Mechanics and Physics of Solids 54(4): 783-806.

Liu, Q., 2006, Literature review: materials with negative Poisson's ratios and potential applications to aerospace and defence, Defence Science and Technology Organisation (DSTO), Department of Defence, Australia, Report \# DSTO-GD-0472.

Ma, H. M., Gao, X.-L. and Reddy, J. N., 2008, A microstructure-dependent Timoshenko beam model based on a modified couple stress theory, Journal of the Mechanics and Physics of Solids 56(12): 3379-3391. 
Miller, W., Mackenzie, D. S., Smith, C. W. and Evans, K. E., 2008, A generalised scaleindependent mechanism for tailoring of thermal expansivity: Positive and negative, Mechanics of Materials 40(4): 351-361.

Palumbo, N. M. A., Smith, C. W., Miller, W. and Evans, K. E., 2011, Near-zero thermal expansivity 2-D lattice structures: Performance in terms of mass and mechanical properties, Acta Materialia 59(6): 2392-2403.

Prawoto, Y., 2012, Seeing auxetic materials from the mechanics point of view: a structural review on the negative Poisson's ratio, Computational Materials Science 58: 140-153.

Roque, C. M. C., Fidalgo, D. S., Ferreira, A. J. M. and Reddy, J. N., 2013, A study of a microstructure-dependent composite laminated Timoshenko beam using a modified couple stress theory and a meshless method, Composite Structures 96: 532-537.

Saxena, K. K., Das, R. and Calius, E. P., 2016, Three decades of auxetics research - materials with negative Poisson's ratio: a review, Advanced. Engineering Materials 2016, DOI: 10.1002/adem.201600053.

Sigmund, O. and Torquato, S., 1996, Composites with extremal expansion coefficients, Applied Physics Letters 69(21): 3203-3205.

Spadaccini, C. M., 2015, Mechanical metamaterials - design, fabrication, and performance, The Bridge 45(4): 28-36.

Steeves, C. A., e Lucato, S. L. D. S., He, M., Antinucci, E., Hutchinson, J. W. and Evans, A. G., 2007, Concepts for structurally robust materials that combine low thermal expansion with high stiffness, Journal of the Mechanics and Physics of Solids 55(9): 1803-1822.

Theocaris, P. S., Stavroulakis, G. E. and Panagiotopoulos, P. D., 1997, Negative Poisson's ratios in composites with star-shaped inclusions: a numerical homogenization approach, Archive of 
Applied Mechanics 67(4): 274-286.

Underhill, R. S., 2014, Defense applications of auxetic materials, DSIAC Journal 1(1): 7-13.

Wei, K., Chen, H., Pei, Y. and Fang, D., 2016, Planar lattices with tailorable coefficient of thermal expansion and high stiffness based on dual-material triangle unit, Journal of the Mechanics and Physics of Solids 86:173-191.

Woodcock, D. A., Lightfoot, P., Villaescusa, L. A., Díaz-Cabañas, M. J., Camblor, M. A. and Engberg, D., 1999, Negative thermal expansion in the siliceous zeolites chabazite and ITQ-4: A neutron powder diffraction study, Chemistry of Materials 11(9): 2508-2514.

Wu, L. L., Li, B. and Zhou, J., 2016, Isotropic negative thermal expansion metamaterials, ACS Applied Materials \& Interfaces 8(27), 17721-17727.

Xu, H. and Pasini, D., 2016, Structurally efficient three-dimensional metamaterials with controllable thermal expansion, Scientific Reports 6: 34924-1 8. DOI:10.1038/srep34924.

Yamamoto, N., Gdoutos, E., Toda, R., White, V., Manohara, H. and Daraio, C., 2014, Thin films with ultra-low thermal expansion, Advanced Materials 26(19): 3076-3080. 